Check for updates

Cite this: J. Mater. Chem. C, 2021, 9, 10591

Received 27th March 2021,

Accepted 26th May 2021

DOI: $10.1039 / \mathrm{d} 1 \mathrm{tc0} 01407 \mathrm{~b}$

rsc.li/materials-c

\section{The quest for single component molecular metals within neutral transition metal complexes $\dagger$}

\begin{abstract}
Mariana F. G. Velho, ${ }^{a b}$ Rafaela A. L. Silva (D) ${ }^{a}$ and Dulce Belo (D) *ac
Twenty years ago, the molecular materials community accomplished a major breakthrough with the report of the first single component molecular metal (SCMM). Previously it was believed that molecular metals had to be composed of a pair of partially oxidized species, a condition necessary to guarantee the formation of the conduction band and the generation of charge carriers. However, by the turn of the millennium a new paradigm arose with the discovery of metallic behaviour in two neutral complexes, [Ni(tmdt) $)_{2}$ (tmdt: trimethylenetetrathiafulvalenedithiolate) and $\left[\mathrm{Au}(\alpha-\mathrm{tpdt})_{2}\right](\alpha-\mathrm{tpdt}$ : 2,3-thiophenedithiolate). Since then, this topic gathered the attention of the scientific community and the number of available SCMM in literature consequentially increased. By their nature, neutral bis(1,2-dithiolene) and bis(1,2-diselenolene) transition metal (BTM) complexes still remain, by far, the best candidates to obtain a SCMM. This review will focus on these materials, their design, synthesis, and properties. Based on their molecular-crystal-band structures, an analysis of the requirements to prepare a SCMM, will also be address.
\end{abstract}

\title{
Introduction
}

${ }^{a}$ Centro de Ciências e Tecnologias Nucleares, Instituto Superior Técnico, Universidade de Lisboa, E.N. 10, P-2695-066 Bobadela LRS, Portugal. E-mail:dbelo@ctn.tecnico.ulisboa.pt

${ }^{b}$ Instituto de Telecomunicações, Instituto Superior Técnico, Av. Rovisco Pais 1, P-1049-001, Lisboa, Portugal

${ }^{c}$ Departament of Nuclear Sciences and Engineering, Instituto Superior Técnico, Universidade de Lisboa, E.N. 10, P-2695-066 Bobadela LRS, Portugal

$\dagger$ This paper is part of a Journal of Materials Chemistry C themed issue in honour of Professor Jaume Veciana and Professor Concepció Rovira.
Molecular metals have been in the spotlight since the 70's, when the first organic metal, TTF-TCNQ (TTF: tetrathiafulvalene, TCNQ: tetracyanoquinodimethane, Chart 1) was announced. ${ }^{1} \mathrm{~A}$ decade later this achievement was surpassed by the discovery of the first organic superconductor, (TMTSF) ${ }_{2} \mathrm{PF}_{6}$ (TMTSF: tetramethyltetraselenafulvalene, Chart 1). ${ }^{2}$ Nowadays hundreds of molecular conductors and superconductors can be found in literature. ${ }^{3,4}$ Most of these molecular salts were prepared using

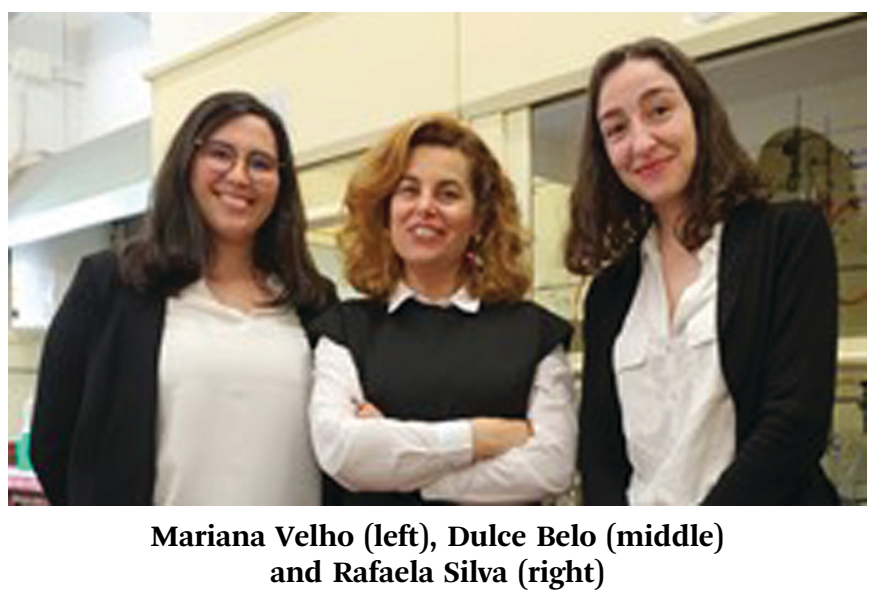

Dulce Belo completed her PhD degree in Chemistry from Instituto Superior Técnico (IST), Universidade de Lisboa, in 2002. She is a researcher at the Solid State Group @IST and has devoted most of her scientific career to the design, synthesis and physical characterization of molecular materials with unconventional magnetic and transport properties. Among them, she developed pioneering work in the preparation and study of Single Component Molecular Metals (SCMM) and currently is interested on their processing as active components for electronic devices.

Rafaela Silva obtained her PhD in Chemistry in 2017 from Instituto Superior Técnico (IST), Universidade de Lisboa, under supervision of Prof. Manuel Almeida. She currently is a research assistant in the Solid State Group@C2TN-IST focusing her research interests in molecular design and synthesis of molecular materials with unconventional electric properties.

Mariana Velho graduated in Chemical Engineering from Instituto Superior de Engenharia de Lisboa in 2015. Currently she is a Chemistry PhD student at Instituto Superior Técnico, Universidade de Lisboa, developing molecular conductors for electronic devices. Her research interests are experimental synthesis and characterization of conducting multifunctional materials. 


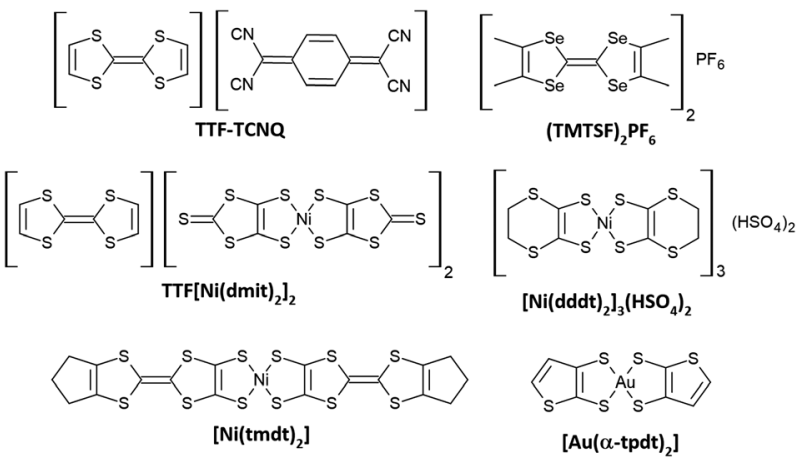

Chart 1 Chemical structures of selected multicomponent and single component molecular materials with metallic or superconducting properties.

TTF derivatives as $\pi$ electron donor molecular units with inorganic anions as the counterpart. In this context, bis(1,2-dithiolene) and bis(1,2-diselenolene) transition metal (BTM) complexes, the inorganic analogues of TTF based molecules, where the central double $\mathrm{C}=\mathrm{C}$ bond is replaced by a transition metal (TM), began to be used as $\pi$ electron acceptor molecular units. Anions such as $\left[\mathrm{M}(\mathrm{dmit})_{2}\right]^{X-}$ (dmit: 4,5-dimercapto-1,3-dithiole-2-dithione) and $\left[\mathrm{M}(\mathrm{mnt})_{2}\right]^{\mathrm{X}-}$ (mnt: maleonitriledithiolene) were widely explored with many of the materials revealing very interesting conducting properties. $^{5-7}$ One of these examples is TTF $\left[\mathrm{Ni}(\mathrm{dmit})_{2}\right]_{2}$ (Chart 1), reported in 1986. It presented a superconducting behaviour with a room temperature conductivity, $\sigma_{\mathrm{RT}}$, of $300 \mathrm{~S} \mathrm{~cm}^{-1}$ and $1.5 \times$ $10^{5} \mathrm{~S} \mathrm{~cm}^{-1}$ at $4.2 \mathrm{~K}^{8}$ BTM complexes can also perform as $\pi$ electron donor molecules, as in $\left[\mathrm{Ni}(\mathrm{dddt})_{2}\right]_{3}\left(\mathrm{HSO}_{4}\right)_{2}$ (dddt: $5,6-$ dihydro-1,4-dithiin-2,3-dithiolate), the first metallic system in the family of $\left[\mathrm{M}(\mathrm{dddt})_{2}\right]$ salts (Chart 1$)^{9}{ }^{9}$

When the advanced materials community realized the important role BTM complexes could play in the solid-state properties, the number of molecular building blocks based on this type of complexes significantly increased, allowing a better understanding of their nature. ${ }^{6}$ As their physical properties began to be understood, it became clear that they were promising candidates to obtain the idealized molecular metal based on a single neutral species. ${ }^{10}$ Until the end of the 20th century, this goal seemed to be distant, since all known molecular conductors persisted to be based on multicomponent molecular crystals, composed by a partially oxidized couple of $\pi$ electron donor/ acceptor molecules. In 2001 the first examples of the so called Single Component Molecular Metals (SCMM) became a reality through the report of $\left[\mathrm{Ni}(\mathrm{tmdt})_{2}\right]$ (tmdt: trimethylenetetrathiafulvalenedithiolate) ${ }^{11}$ and $\left[\mathrm{Au}(\alpha \text {-tpdt })_{2}\right]$ ( $\alpha$-tpdt: 2,3-thio-

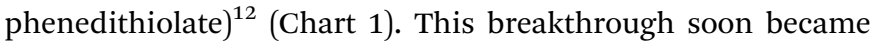
the new hot topic in materials science. The advantage of SCMM relatively to other traditional molecular metals is the simplicity of involving just one neutral molecule to be designed and prepared, which may also help to minimise polymorphism observed in the solid-state. Relatively to typical elemental metals, SCMM offer the possibility of being multifunctional, since in the same material several physical properties can be combined. If properly designed, they can be soluble allowing their processability as lighter and transparent components for electronic devices. With the exception of a pure organic SCMM based on a TTF derivative, a zwitterionic TTF-extended dicarboxylate radical (TED), ${ }^{13}$ all reported examples of SCMM are neutral BTM complexes. This review will focus on those studied as SCMM. In this perspective their design, synthesis, crystal structures and correlation with their transport and magnetic properties will be addressed.

\section{Bis(1,2-dithiolene) and bis(1,2-diselenolene) transition metal (BTM) complexes}

Since this review will be dedicated to SCMM based on neutral BTM complexes, it becomes relevant to address some general features of these neutral complexes before proceeding on the SCMM state of the art discussion.

BTM complexes are often considered the inorganic analogues of TTF derivatives,${ }^{14}$ having two bidentate ligands, 1,2-dithiolate or 1,2-diselenolate ligands, that coordinate to the metal, respectively by the sulphur or selenium atoms (Chart 2). In this review only homoleptic BTM complexes will be addressed.

\section{Electronic and structural properties}

The electronic structure of 1,2-dithiolate or 1,2-diselenolate ligands impacts on the properties revealed by the BTM complexes, since the ligands often play a non-innocent role. The organic ligand forms a planar five member ring with the metal centre with such electronic flexibility that, in most situations, tolerates the variation of the redox state of the complex without changing the molecular geometry, ${ }^{15}$ making accessible redox oxidation states ranging from -2 to +1 . The determination of the oxidation state of BTM complexes is not always obvious due to the non-innocent character of the ligand, with the electron density, not mainly localized in the central metal atom, but instead distributed all over the atoms of the molecule. ${ }^{15}$ Nevertheless, important information can be obtain through an analysis of their bond lenghts and angles. ${ }^{15}$

The M-S bond ranges between $2.101 \AA$ and $2.563 \AA$, with Ni complexes exhibiting the smaller average bond length. For complexes with the same ligand and metal centre, the typical rule is "the smaller the oxidation state is, the longer will be M-S bond lengths". ${ }^{15}$ The other bond lengths and angles averages also show some uniformity. ${ }^{15,16}$

Different models have been used to explain the electronic structure of BTM complexes, ${ }^{17-19}$ but nowadays the community accepts that each family of BTM complexes behaves as an unique system and that to fully understand their proporties a detailed electronic density mapping is necessary.

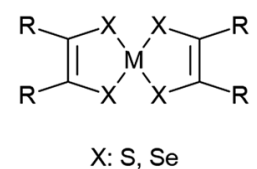

Chart 2 General chemical structure of bis(1,2-dithiolene) (X: S) and bis(1,2-diselenolene) (X: Se) complexes, $\left[M\left(X_{2} C_{2} R_{2}\right)_{2}\right](X: S, S e)$. 
BTM complexes usually present a square planar or near tetrahedral coordination geometry around the metal centre, with the dihedral angle between the two chalcogen-metal-chalcogen planes ranging between $0-10^{\circ}$ and $80-90^{\circ}$, respectively. Rare cases with a square pyramidal geometry were also found. ${ }^{15}$

In molecular materials the crystal packing is crucial to their solid-state properties, where the number and type of intermolecular interactions are very important. It is experimentally recognized that S $\cdots$ S and Se. -Se short contacts are fundamental to increase the dimensionality of the system. Charge assisted hydrogen bonds and strong $\pi \cdots \pi$ interactions are often also structuring, contributing to the consolidation of the crystal structure. The lack of bulky or electron deficient $\pi$ substituents outside of the organic ligand plane can potentiate important metal-chalcogen or metal-metal interactions between the molecular units leading to well-defined multimeric structures, such as dimers and trimers. ${ }^{6,15}$

\section{Synthesis}

Apart from some specific cases, the majority of precursors used to prepare BTM complexes are 1,3-dithiole-2-chalcogenones or 1,3-diselenole-2-chalcogenones and asymmetric TTFs with terminal sulphur cyanoethyl groups (Scheme 1). ${ }^{12,20}$ The cyanoethyl groups can also be anchored to different frameworks and then undergo the same synthetic route to prepare the correspondent BTM complexes. $^{21}$

The synthesis of 1,3-dithiole-2-chalcogenones have many possible routes. ${ }^{22}$ These chalcogenones can be use, directly as ligand precursors in situ, by hydrolytic cleavage with sodium methoxide, under inert atmosphere. Then the appropriate metal is added, usually as a metallic halide. The complex in its anionic form is then precipitated from the solution, as tetraalkylammonium or tetraphenylphosphonium salts (Scheme 1). ${ }^{23}$ When electrochemically accessible, the neutral complex is obtained by chemical oxidation (e.g. iodine or air exposure $)^{23}$ of the anionic species or through electrocrystallisation techniques, in the appropriate organic solvent and a supporting electrolyte, such as $n \mathrm{Bu}_{4} \mathrm{~N}\left(\mathrm{ClO}_{4}\right)$ and $n \mathrm{Bu}_{4} \mathrm{~N}\left(\mathrm{PF}_{6}\right)$. The electrocrystallisation is carried out at a controlled temperature in an $\mathrm{H}$-type cell, using two platinum electrodes and applying a constant current, usually around $0.1-0.5 \mu \mathrm{A},{ }^{20}$ although higher currents can also be used if necessary.

The dmit ligand is prepared through a different route by the reduction of carbon disulphide with sodium and then it is separated from the mixture in the form of $\left(\mathrm{Et}_{4} \mathrm{~N}\right)_{2}\left[\mathrm{Zn}(\mathrm{dmit})_{2}\right] \cdot{ }^{24}$ This zinc complex is usually used to prepare not only other

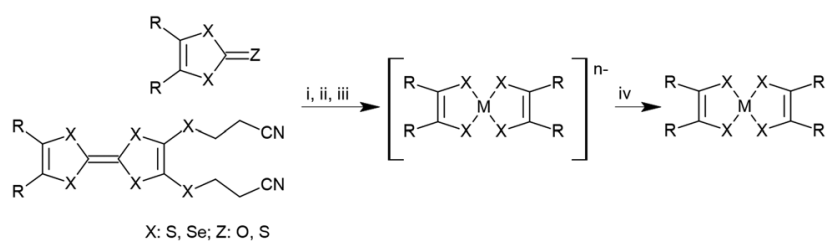

Scheme 1 General synthetic route to obtain anionic and neutral BTM complexes: (i) $\mathrm{MeONa}$ in $\mathrm{MeOH}$; (ii) $\mathrm{NiCl}_{2} \cdot 6 \mathrm{H}_{2} \mathrm{O}, \mathrm{CuCl}_{2} \cdot 2 \mathrm{H}_{2} \mathrm{O}, \mathrm{KAuCl}_{4}$, $\mathrm{PdCl}_{2}(\mathrm{PhCN})_{2}$ or $\mathrm{CoCl}_{2} \cdot 6 \mathrm{H}_{2} \mathrm{O}$ in $\mathrm{MeOH}$; (iii) (alkyl) ${ }_{4} \mathrm{NBr}$ or $\mathrm{PPh}_{4} \mathrm{Br}$ in $\mathrm{MeOH}$; (iv) electrocrystallisation, air exposure or $\mathrm{I}_{2}$.
$\left[\mathrm{M}(\mathrm{dmit})_{2}\right]$ complexes, but also the 4,5-bis(2'-cyanoethylthio)1,3-dithiole-2-thione. This compound has the reactive sulphur atoms protected by cyanoethyl groups ${ }^{25}$ and it is used to prepare asymmetric TTFs via cross-coupling reactions with chalcogenones mediated by trialkyl phosphite. ${ }^{20}$ An alternative route is a pseudo-Wittig condensation, ${ }^{20}$ generally used to synthesise TTFs with alkyl substituents. In both cases, the molecules obtained are the precursors for BTM complexes with extended TTF moieties. Besides the route already illustrated in Scheme $1,{ }^{26}$ the cyanoethyl protecting groups can be removed in a methanol solution of $\mathrm{Me}_{4} \mathrm{~N}(\mathrm{OH})$ or $n \mathrm{Bu}_{4} \mathrm{~N}(\mathrm{OH})$ creating the coordination sites. Then, a solution of the metal precursor is added at $-78{ }^{\circ} \mathrm{C}$ and after the mixture is gradually warmed to room temperature, creating the anionic complex, the corresponding neutral complex can then also be obtained by chemical oxidation or electrocrystallisation techniques. $^{20}$

All these synthetic procedures must be carried out under strictly inert conditions because the anionic species of the metal complex is often very sensitive to oxygen and readily oxidised to the neutral state.

\section{Single component molecular metals (SCMM) and conductors}

The first neutral BTM complexes displaying conducting properties were discovered around $1996 .^{27,28}$ However, only in 2001 the first SCMM, based on this type of materials, were reported. In the following sections, the requirements for designing SCMM will be discussed, and the solid-state properties will be addressed based on the most relevant examples of single component molecular conductors (SCMC) and SCMM.

Prior to SCMM presenting metallic behaviour a molecular material needed to be composed of at least two components (an electronic $\pi$ donor and an acceptor) and fulfil two specific requirements: (1) the formation of a conduction band and (2) the generation of charge carriers. These two conditions are also necessary to obtain a SCMM. The first requirement is achieved by means of strong intermolecular interactions, with significant transverse interactions, to obtain 2D or 3D systems, leading to compact crystal structures which allows the formation of wide overlapping bands. The second requirement was the true challenge in preparing SCMM. ${ }^{14}$ In the case of molecular metals based on a neutral species, the electrons transfer can only occur internally, between the HOMO (highest occupied molecular orbital) and LUMO (lowest unoccupied molecular orbital), often separated by a significant gap. If the molecule has an even number of electrons, this would result in a completely filled HOMO precluding the generation of charges. ${ }^{14}$ But if the molecule has an odd number of electrons, the material would behave as a Mott-insulator.

So how do SCMM overcome this obstacle?

By designing molecules with a small gap between the HOMO and LUMO. The first theoretical models used to describe the conducting mechanism in SCMM and conductors, considered a parallel arrangement of the bands. In this case the HOMO-LUMO 


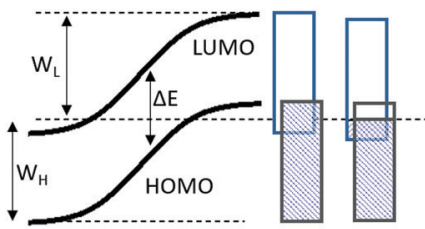

Parallel Bands

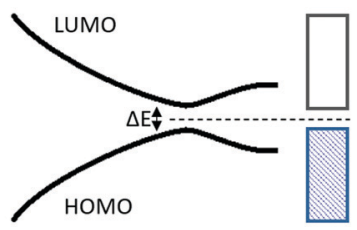

Crossing Bands
Fig. 1 Schematic representation of parallel and crossing band structures, where $\Delta E$ is the HOMO-LUMO gap and $w_{H}$ and $w_{L}$ are the widths of the HOMO and LUMO bands, respectively. Adapted from ref. 29 with permission from The Royal Society of Chemistry.

overlap would allow the transfer of electrons between them resulting in bands partially filled, leading to a semimetallic system (Fig. 1). ${ }^{29}$ Nevertheless, this situation is very rare. A crossing band structure is the most common case (Fig. 1), which occurs when the interaction between the HOMO and LUMO results in the opening of a gap at the Fermi level. In these cases the dimensionality of the systems is very important, since $2 \mathrm{D} / 3 \mathrm{D}$ large transversal interactions can overcome or reduce the gap and stabilize the metallic state. ${ }^{14}$

More recently some SCMM and conductors were better described as Dirac electron systems (Fig. 2), ${ }^{30-34}$ which may be seen as a very particular situation of a crossing band structure. In a Dirac electron system, the HOMO and LUMO bands cross at Dirac points, at variance with the characteristic gap opening in the crossing band structure. This feature confers a semimetallic behaviour to these systems.

BTM complexes are good candidates to obtain SCMM because they have similar frontier orbitals to the HOMO of TTF based molecular conductors (Fig. 3). ${ }^{20}$ In addition, in this type of molecules the gap between HOMO and LUMO is already known to be small and it can be further reduced in the solid state through the enhancement of intermolecular interactions. BTM complexes also have unlimited possibilities to tune the designing of their ligands and accommodate chalcogen atoms or TTF moieties. These two advantages have been studied and explored consistently and it is known that in the first case chalcogens atoms have the same sign of the overlap integral in every sulphur and selenium atoms and tend to form $\mathrm{S}(\mathrm{Se}) \cdots \mathrm{S}(\mathrm{Se})$ short contacts both along the

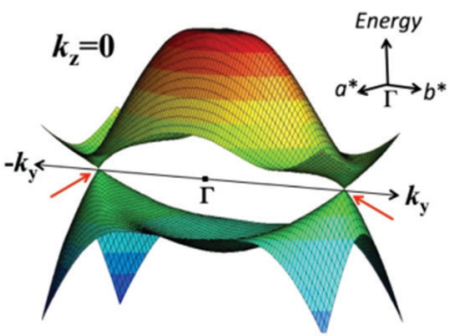

Fig. 2 Band structure of $\left[\mathrm{Pd}(\mathrm{dddt})_{2}\right]$ at $8 \mathrm{GPa}$, with the Dirac cones indicated by red arrows. Adapted with permission from Kato, R., Cui, H., Tsumuraya, T., Miyazaki, T., \& Suzumura, Y. (2017). Emergence of the Dirac electron system in a single-component molecular conductor under high pressure. J. Am. Chem. Soc., 139(5), 1770-1773. DOI: 10.1021/jacs.6b12187. Copyright 2017 American Chemical Society.

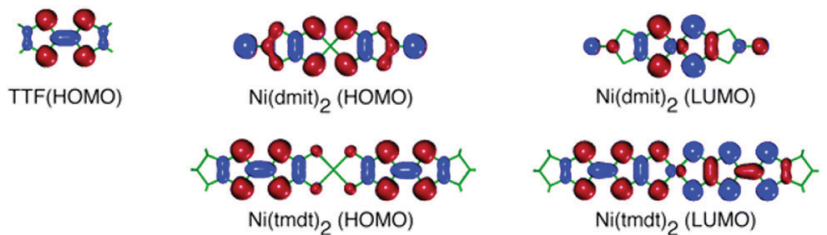

Fig. 3 Schematic representation of the HOMO and LUMO of TTF and BTM complexes $\left[\mathrm{Ni}(\mathrm{dmit})_{2}\right]$ and $\left[\mathrm{Ni}(\mathrm{tmdt})_{2}\right]$. Adapted with permission from Kobayashi, A., Fujiwara, E., \& Kobayashi, H. (2004). Single-Component Molecular Metals with Extended-TTF Dithiolate Ligands. Chem. Rev., 104(11), 5243-5264. DOI: 10.1021/cr030656l. Copyright 2004 American Chemical Society.

direction of the stacking and also transversely. ${ }^{14}$ The incorporation of TTFs in the ligand core leads to a larger delocalisation of $\pi$ electrons along the molecule. ${ }^{14}$ Also, depending on the central TM, the molecule can have an odd or even number of electrons.

The next sections will focus on the solid-state properties of the most relevant BTM complexes, studied in the scope of SCMC.

\section{BTM complexes with extended TTF units}

Narvor et $a l^{28}$ was a pioneer in exploring neutral BTM complexes comprising TTF moieties. The family of neutral $\left[\mathrm{M}(\mathrm{dttfdt})_{2}\right]$ complexes (dttfdt: dithioalkyltetrathiafulvalenedithiolate; $\mathrm{M}: \mathrm{Hg}$ with R: Et; M: Ni with R: Me, Et, Bu, Chart 3) was the first reported. The neutral $\left[\mathrm{Hg}(\mathrm{dttfdt})_{2}\right](\mathrm{R}: \mathrm{Et})^{35,36}$ revealed a room temperature conductivity, $\sigma_{\mathrm{RT}}$, measured in compressed pellets of $10^{-5} \mathrm{~S} \mathrm{~cm}^{-1}$. The methyl, ethyl, and butyl derivatives of $\left[\mathrm{Ni}(\mathrm{dttfdt})_{2}\right]$ displayed a $\sigma_{\mathrm{RT}}$, also measured in compressed pellets, of $10^{-1} \mathrm{~S} \mathrm{~cm}^{-1}$ $\left(E_{\mathrm{a}}=0.09 \mathrm{eV}\right), 2.7 \times 10^{-2} \mathrm{~S} \mathrm{~cm}^{-1}\left(E_{\mathrm{a}}=0.10 \mathrm{eV}\right)$ and $10^{-4} \mathrm{~S} \mathrm{~cm}^{-1}$ $\left(E_{\mathrm{a}}=0.23 \mathrm{eV}\right)$, respectively. ${ }^{28,35}$ All complexes show a semiconducting behaviour, except the methylated [Ni(dttfdt $)_{2}$ ] complex, which above $275 \mathrm{~K}$ behaves as a metal, while displaying semiconducting behaviour below this temperature. ${ }^{35}$

The $\left[\mathrm{Pd}(\mathrm{C} 3 \text {-tdt })_{2}\right]$ (C3-tdt: di- $n$-propylthiotetrathiafulvalenedithiolate, Chart 3) complex was reported a few years later. Its transport properties, measured in single crystal, revealed a semiconductor behaviour with a $\sigma_{\mathrm{RT}}$ of $10^{-2} \mathrm{~S} \mathrm{~cm}^{-1}\left(E_{\mathrm{a}}=0.034 \mathrm{eV}\right.$ at $300-120 \mathrm{~K}$ and $0.011 \mathrm{eV}$ at $120-80 \mathrm{~K}) .^{37}$

Following this path, Kobayashi and co-workers, managed to prepare the first family of SCMM, the $\left[\mathrm{M}(\mathrm{tmdt})_{2}\right]$ (tmdt: trimethylenetetrathiafulvalenedithiolate, R: Ni, Au, Pt, Zn, Cu, Co and $\mathrm{Pd}$, Chart 3). The first reported example was the nickel complex $\left[\mathrm{Ni}(\mathrm{tmdt})_{2}\right]$, where the planar neutral molecules crystallise in a very compact pattern composed by domino-like chains connected by a 3D array of transverse $\mathrm{S} \cdot \mathrm{S}$ short contacts (3.350-3.675 ̊̊) (Fig. 4). ${ }^{11,29}$

Single crystal resistivity measurements showed a $\sigma_{\mathrm{RT}}$ of $400 \mathrm{~S} \mathrm{~cm}^{-1}$ (200 S cm$~^{-1}$ in compressed pellet) and a metallic behaviour down to $0.6 \mathrm{~K} .{ }^{11}$ The magnetic properties were also studied and the complex revealed a Pauli-like paramagnetic behaviour with a magnetic susceptibility, $\chi$, of $3.1 \times 10^{-4} \mathrm{emu} \mathrm{mol}^{-1}$ at room temperature and $2.7 \times 10^{-4} \mathrm{emu} \mathrm{mol}^{-1}$ at $2 \mathrm{~K}$, showing an approximately temperature-independent $\chi$. The results are compatible with the properties of a metallic system. ${ }^{11}$ The existence of Fermi surfaces in $\left[\mathrm{Ni}(\mathrm{tmdt})_{2}\right]$ was experimentally corroborated through measurements of the Haas-van Alphen (dHvA) oscillations, 
Extended-TTF

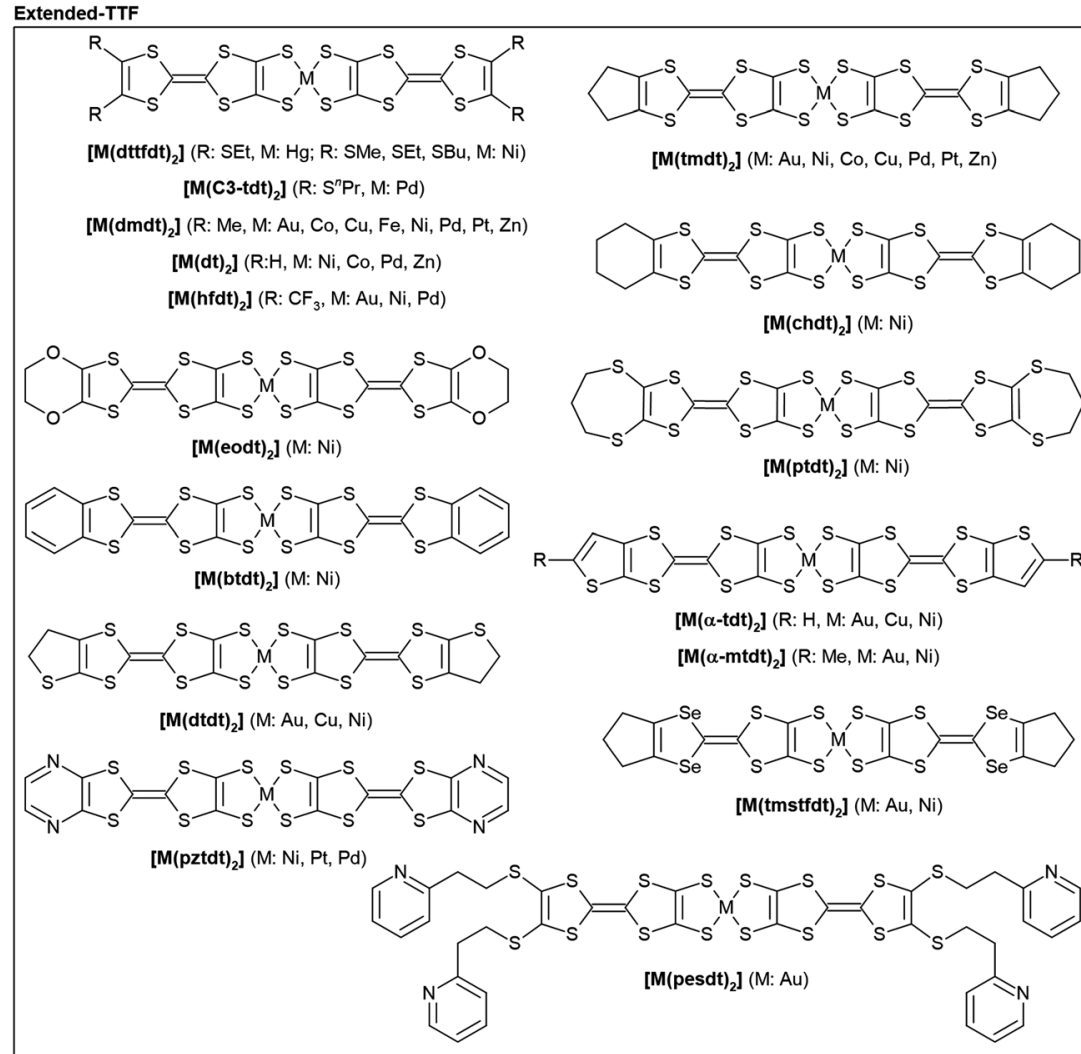

Thiazole

[M(R-thiazdt) $)_{2}$ (R: Me, Et, EtOH, $\mathrm{NMe}_{2}, i-\mathrm{Pr}, c-\mathrm{Pr}, n-\mathrm{Pr}, \mathrm{M}: \mathrm{Au}$;

$\mathrm{R}: \mathrm{Me}, \mathrm{Et}, \mathrm{M}: \mathrm{Ni}, \mathrm{Pt}$ )<smiles></smiles>

[M(R-thiazodt)] ] (R: Et, M: Au)

$R S-\left\langle\prod_{S}^{N} \prod_{S}^{S} M_{S}^{S} \prod_{N}^{S}\right\rangle-S R$

[M(RS-tzdt) $)_{2}$ (R: Me, Et, EtOH,

$\mathrm{M}: \mathrm{Au}, \mathrm{Ni})$

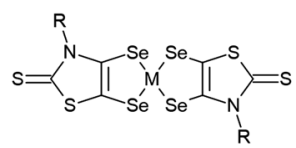

$\left[M(R-\text { thiazds) })_{2}\right.$ (R: Et, M: Au)

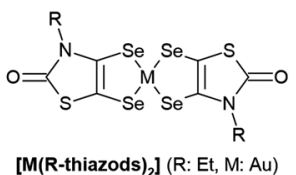

$\left[M(R-\text { thiazods) })_{2}\right.$ (R: Et, M: Au)

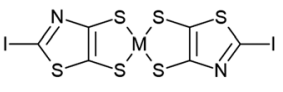

[M(I-tzdt) $)_{2}$ (M: Au)

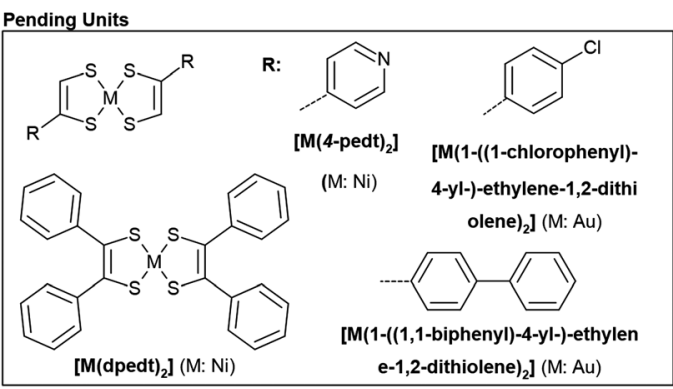

Thiophene/Selenophene

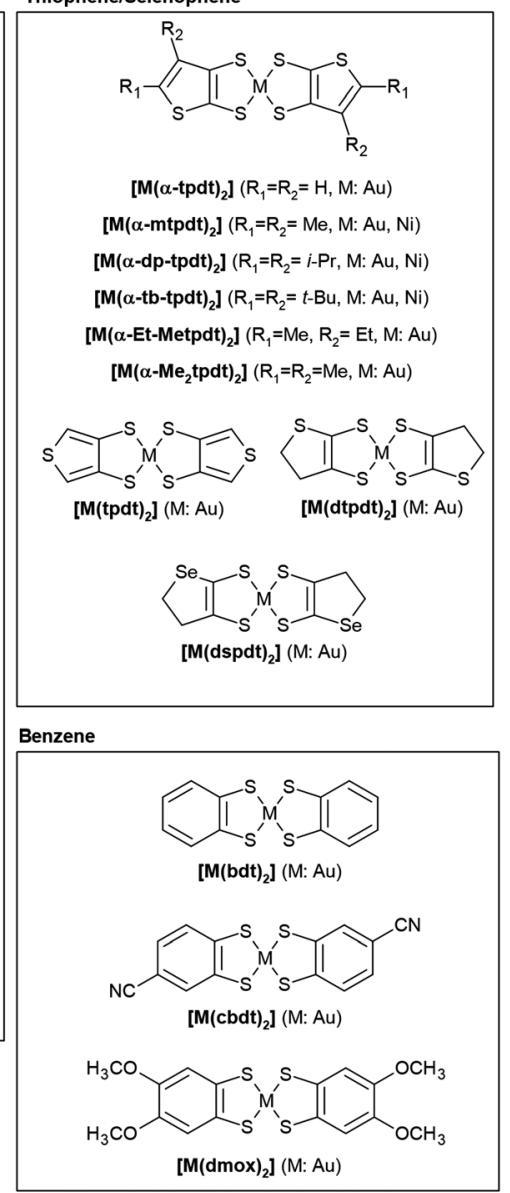

Other S/Se-Containing Heterocycles

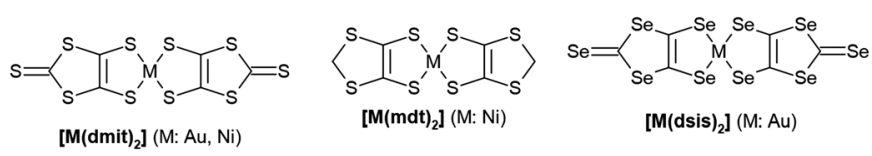
[M(dddt)] (M: Au, Ni, Pd, Pt) $\begin{gathered}\text { [M(medt) })_{2}(\mathrm{R}: \mathrm{Me}, \mathrm{M}: \mathrm{Ni}) \\ {\left[\mathrm{M}(\mathrm{phdt})_{2}\right](\mathrm{R}: \mathrm{Ph}, \mathrm{M}: \mathrm{Ni})}\end{gathered}$

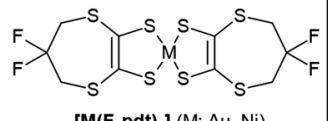

$\left[\mathrm{M}\left(\mathrm{F}_{2} \mathrm{pdt}\right)_{2}\right](\mathrm{M}: \mathrm{Au}, \mathrm{Ni})$
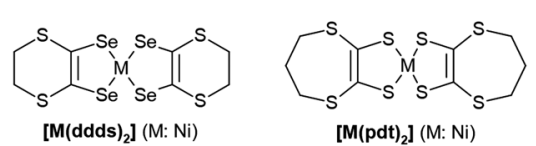
[M(pdt) $\left.)_{2}\right](\mathrm{M}: \mathrm{Ni})$
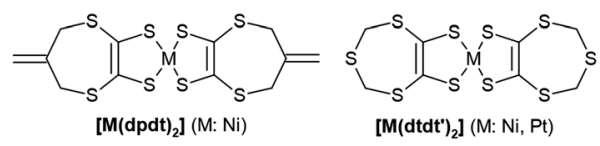

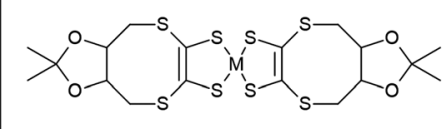

[M(diotte) $)_{2}$ (M: Ni)

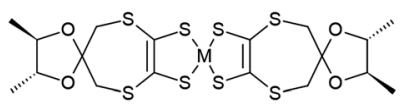

[M(chiral 4,5-dimethyl-1,3-dioxolane substituted pdt $)_{2}$ ] (M: Au)

Chart 3 Neutral BTM complexes prepared and studied in the context of SCMM or conductors.

which showed a clear dHvA oscillation in all directions confirming the 3D metalllic behaviour. ${ }^{14,38}$
Within the isostructural neutral tmdt family of complexes (M: $\mathrm{Ni}, \mathrm{Au}, \mathrm{Cu}, \mathrm{Pd}$ and $\mathrm{Pt}$ ) the transport properties were 

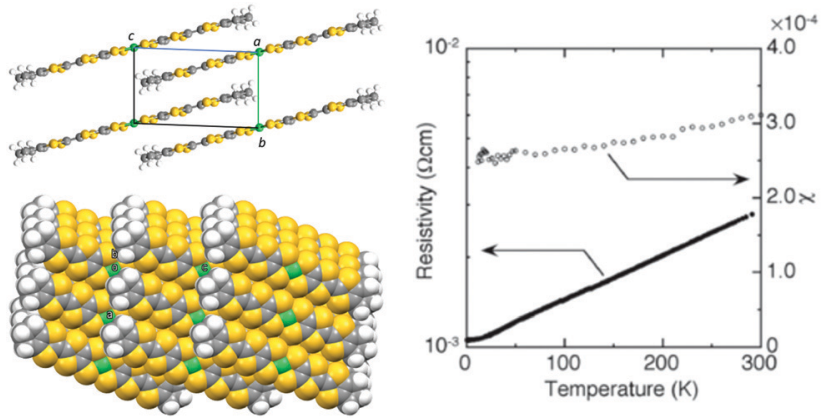

Fig. 4 Left: Details showing the domino-like molecular overlapping and the 3D compact molecular packing in the crystal structure $\left[\mathrm{Ni}(\mathrm{tmdt})_{2}\right]$ Right: Temperature dependence of resistivity and magnetic susceptibility $\left(\chi\right.$, emu $\left.\mathrm{mol}^{-1}\right)$ of $\left[\mathrm{Ni}(\mathrm{tmdt})_{2}\right]$. From Tanaka, H., Okano, Y., Kobayashi, H., Suzuki, W., \& Kobayashi, A. (2001). A Three-Dimensional Synthetic Metallic Crystal Composed of Single-Component Molecules. Science, 291(5502), 285-287. DOI: 10.1126/science.291.5502.285. Reprinted with permission from AAAS.

measured in polycrystalline pellets, with exception of the Pd and $\mathrm{Ni}$ complexes, that were measured as single crystals. The gold complex transport properties showed an increase of resistivity with decreasing temperature, nevertheless with a high $\sigma_{\mathrm{RT}}$ of $50 \mathrm{~S} \mathrm{~cm}{ }^{-1} \cdot{ }^{14,39,40}$ The magnetic properties measurements revealed a $\chi_{\mathrm{RT}}$ of $4.2 \times 10^{-4} \mathrm{emu} \mathrm{mol}^{-1}$ and also exposed an antiferromagnetic transition at $110 \mathrm{~K}$, despite the remaining relatively high conductivity of $\left[\mathrm{Au}(\mathrm{tmdt})_{2}\right]$ at this temperature $\left(20 \mathrm{~S} \mathrm{~cm}^{-1}\right){ }^{14,39,40}$ In the case of $\left[\mathrm{Pt}(\mathrm{tmdt})_{2}\right]$, a metallic behaviour was observed down to the liquid-helium temperature, with a $\sigma_{\mathrm{RT}}$ of $350 \mathrm{~S} \mathrm{~cm}^{-1}$ and an even higher $\sigma_{4 \mathrm{~K}}$, of approximately $550 \mathrm{~S} \mathrm{~cm}^{-1}$ (Fig. 5). ${ }^{41}$ This metallic behaviour is achieved not only through the contribution of very short transverse $\mathrm{S} \cdots \mathrm{S}$ intermolecular contacts (3.348-3.643 $\AA$ ), even shorter than those found in [Ni(tmdt $\left.)_{2}\right]$, but also due to the efficient $\pi \cdots \pi$ overlapping, conferring a strong $3 \mathrm{D}$ nature to the system. ${ }^{41}$ The magnetic susceptibility measurements revealed a $\chi_{\mathrm{RT}}$ of $\sim 1.5 \times 10^{-4} \mathrm{emu} \mathrm{mol}^{-1}$ that slowly decreases upon cooling until $170 \mathrm{~K}$ where it stabilizes at $\sim 1.3 \times 10^{-4} \mathrm{emu} \mathrm{mol}^{-1}$ (Fig. 5), resembling a Pauli-like paramagnetic behaviour, also consistent with its metallic behaviour. ${ }^{41}$

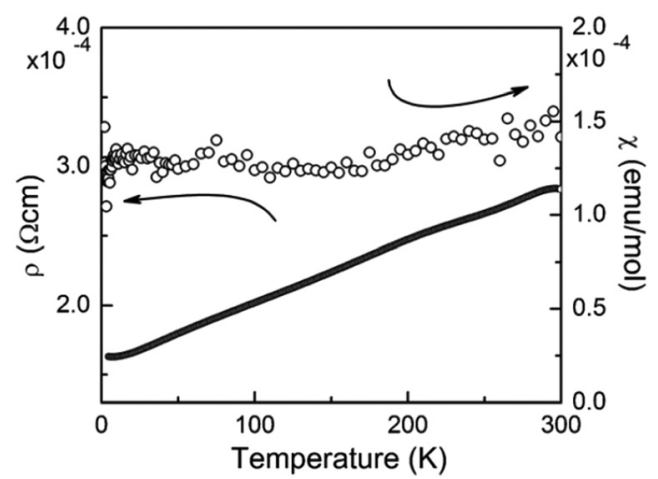

Fig. 5 Temperature dependence of resistivity $(\rho)$ and magnetic susceptibility $(\chi)$ of $\left[\mathrm{Pt}(\mathrm{tmdt})_{2}\right]$ measured in a compressed polycrystalline sample. Reprinted with permission from ref. 41. Copyright (C)2009 Wiley-VCH Verlag GmbH \& Co. KGaA, Weinheim.
$\left[\mathrm{Cu}\left(\mathrm{tmdt}_{2}\right)_{2}\right.$ displayed a $\sigma_{\mathrm{RT}}$ around $7 \mathrm{~S} \mathrm{~cm}^{-1}$ and a semiconducting behaviour, but under pressure the conductivity increases linearly, suggesting a metallic character. It also behaves as a $1 \mathrm{D}$ antiferromagnetic Heisenberg system, with magnetic ordering at $13 \mathrm{~K}^{42}$ In turn, the $\left[\mathrm{Pd}(\mathrm{tmdt})_{2}\right]$ behaves as a semimetal around room temperature $\left(\sigma_{\mathrm{RT}}=100 \mathrm{~S} \mathrm{~cm}^{-1}\right)$ but a semiconducting behaviour arises as the temperature drops, although its band structure resembles the $\mathrm{Ni}$ and Pd analogues. Moreover, it is the only conductor with an even number of electrons that shows evidences of an antiferromagnetic ordering. ${ }^{43}$

The relevance of the metal $d$ valence orbitals on the formation of the frontier orbitals and the conduction bands was understood upon the synthesis of $\left[\mathrm{Zn}(\mathrm{tmdt})_{2}\right]$ complex, since zinc has all $3 d$ orbitals fully occupied. The neutral [ $\left.\mathrm{Zn}(\mathrm{tmdt})_{2}\right]$ molecules revealed a tetrahedral geometry around the metal atom with both ligands planar and in planes almost perpendicular to each order. Its crystal structure also shows neighbouring molecules overlapping in a face-to-face fashion. This complex displayed in a compressed pellet a semiconducting behaviour with a $\sigma_{\mathrm{RT}}$ of $2 \times 10^{-2} \mathrm{~S} \mathrm{~cm}^{-1}\left(E_{\mathrm{a}}=150 \mathrm{meV}\right)$. The extended-Hückel tight-binding band structure calculation of $\left[\mathrm{Zn}(\mathrm{tmdt})_{2}\right]$ showed that the HOMO and LUMO are almost degenerated due to the orthogonality of the two ligands, which means that they do not contribute to the formation of the 3D conduction band. ${ }^{14,44}$

[Ni(dmdt) $)_{2}$ (dmdt: dimethyltetrathiafulvalenedithiolate, Chart 3) resembles in many ways $\left[\mathrm{Ni}\left(\mathrm{tmdt}_{2}\right)_{2}\right.$, since they both share the same type of crystalline structure. ${ }^{20}\left[\mathrm{Ni}\left(\mathrm{dmdt}_{2}\right]\right.$, measured in a compressed pellet, displays a $\sigma_{\mathrm{RT}}$ of $300-400 \mathrm{~S} \mathrm{~cm}^{-1}$ and a $\sigma_{4 \mathrm{~K}}$ of $150 \mathrm{~S} \mathrm{~cm}^{-1} \cdot{ }^{29} \mathrm{~A}$ behaviour typical of metallic systems can be observed down to $230 \mathrm{~K}$. With no structural data for $\left[\mathrm{Au}(\mathrm{dmdt})_{2}\right]$ its transport properties, measured in a compressed pellet, revealed a semiconductor behaviour down to $50 \mathrm{~K}$, with a $\sigma_{\mathrm{RT}}$ of $12 \mathrm{~S} \mathrm{~cm}^{-1}$ and a very low $E_{\mathrm{a}}$ of $9 \mathrm{meV}^{40}$ However, the magnetic susceptibility measurements suggest a metallic behaviour, showing a $\chi_{\mathrm{RT}}$ of $3.4 \times$ $10^{-4} \mathrm{emu} \mathrm{mol}^{-1}$ and a Pauli-like behaviour down to $50 \mathrm{~K}^{40}$

The crystal structure of $\left[\mathrm{Cu}(\mathrm{dmdt})_{2}\right]$ is similar to $\left[\mathrm{Zn}(\mathrm{tmdt})_{2}\right]$, with a tetrahedral molecular geometry, where each one of the ligands also overlaps face-to-face with a ligand in neighbouring molecules, forming a compact molecular packing of non-planar molecules (Fig. 6). The crystal structure resembles the $\kappa$-type seen in 2D organic superconductors, converting $\left[\mathrm{Cu}(\mathrm{dmdt})_{2}\right]$ in the first 3D $\kappa$-type molecular conductor. ${ }^{14,45}$ This neutral $\mathrm{Cu}$ complex shows a near Curie-Weiss behaviour, with $\chi_{\mathrm{RT}}$ of $1.09 \times 10^{-3} \mathrm{emu} \mathrm{mol}^{-1}$, pointing to the existence of an isolated $\mathrm{S}=1 / 2$ spin on each molecule. Above $100 \mathrm{~K}, \chi_{T}$ is linear

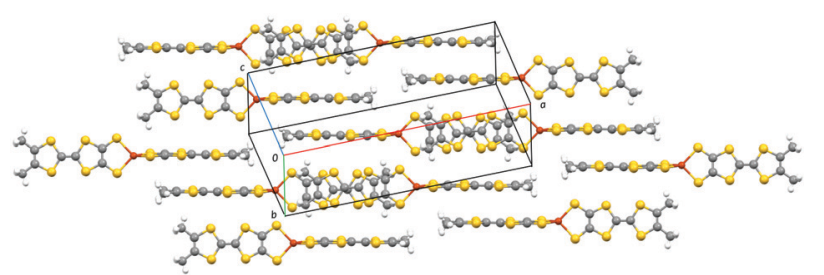

Fig. 6 Detail showing the overlapping mode in the crystal structure of $\left[\mathrm{Cu}(\mathrm{dmdt})_{2}\right]$. 
suggesting a Pauli-like behaviour $\left(\chi_{\mathrm{p}}=3.7 \times 10^{-4} \mathrm{emu} \mathrm{mol}^{-1}\right) .{ }^{14,45}$ Single crystal resistivity measurements revealed a $\sigma_{\mathrm{RT}}\left(110 \mathrm{~S} \mathrm{~cm}^{-1}\right)$ and a weak metallic behaviour around room temperature, with a transition to an insulating state around $95 \mathrm{~K}$. However, this transition is supressed at $3.3 \mathrm{GPa}^{46}$

Other $\left[\mathrm{M}(\mathrm{dmdt})_{2}\right]$ complexes were also studied, such as palladium, ${ }^{20,47}$ platinum, ${ }^{32}$ iron, ${ }^{48}$ zinc $^{44}$ and cobalt ${ }^{49}$ analogues. The $\left[\mathrm{Zn}(\mathrm{dmdt})_{2}\right]$ displays a behaviour similar to $\left[\mathrm{Zn}(\mathrm{tmdt})_{2}\right],{ }^{44}$ while the $\left[\mathrm{Fe}(\mathrm{dmdt})_{2}\right]$ and $\left[\mathrm{Co}(\mathrm{dmdt})_{2}\right]$ complexes are dimerised and revealed lower conductivities and semiconducting behaviours. ${ }^{44,48}$

In turn, the $\left[\mathrm{Pt}(\mathrm{dmdt})_{2}\right]$ measured in a compressed pellet,

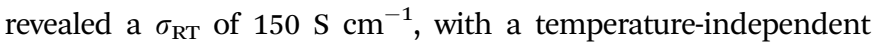
resistivity, showing a semimetallic behaviour, but a temperaturedependent magnetic susceptibility. The band calculations indicate that $\left[\mathrm{Pt}(\mathrm{dmdt})_{2}\right]$ is an ambient-pressure molecular Dirac electron system, also described as a nodal line semimetal. ${ }^{32,34}$

Two other reported examples were $\left[\mathrm{Ni}(\mathrm{chdt})_{2}\right]$ (chdt: cyclohexenotetrathiafulvalenedithiolate) and $\left[\mathrm{Ni}(\text { eodt })_{2}\right]$ (eodt: ethylenedioxytetrathiafulvalenedithiolate) (Chart 3), with $\sigma_{\mathrm{RT}}$ measured on compressed pellets ranging from 1 to $10 \mathrm{~S} \mathrm{~cm}^{-1} \cdot{ }^{14,20,50}$ In the case of $\left[\mathrm{Ni}(\text { eodt })_{2}\right]$, the resistivity decreases until $120 \mathrm{~K}$, suggesting a metallic behaviour, but then it increases slowly, although retaining a reasonably conductivity at $0.6 \mathrm{~K} .{ }^{14,20,50}$

The following example was prepared aiming at obtaining neutral complexes more soluble in common organic solvents, whereby their molecular structure contains solubilizing chains or polar groups. ${ }^{51}$ However, in the $\left[\mathrm{M}(\mathrm{hfdt})_{2}\right]$ family (hfdt: bis(trifluoromethyl)tetrathiafulvalenedithiolate, $\mathrm{M}$ : $\mathrm{Au}, \mathrm{Ni}$ and Pd, Chart 3), ${ }^{51-53}$ the solubility was not improved, since the F . - F segregation effect led to crystal structures composed of $2 \mathrm{D}$ layers separated by $\mathrm{CF}_{3}$ groups. In the case of the $\mathrm{Pd}$ complex, the neutral molecules are dimerised through a metal-metal bond (3.067 Å, Fig. 7a), giving rise to a diamagnetic behaviour. Consequently, the electric conductivity measurements showed in all cases a semiconducting behaviour with $\sigma_{\mathrm{RT}}$ of $1.6 \times 10^{-3} \mathrm{~S} \mathrm{~cm}^{-1}, 3.2 \times$ $10^{-3} \mathrm{~S} \mathrm{~cm}^{-1}$ and $3.7 \times 10^{-3} \mathrm{~S} \mathrm{~cm}^{-1}$ for the Ni, Au and Pd neutral complexes, respectively. ${ }^{51,53}\left[\mathrm{Ni}(\mathrm{hfdt})_{2}\right]$ was submitted to resistivity measurements up to $10 \mathrm{GPa}$, which showed a decreasing of resistivity with pressure. At 7.5 GPa, the activation energy was zero, and at 7.5-8.6 GPa a superconducting transition was observed (Fig. 8). ${ }^{54}$

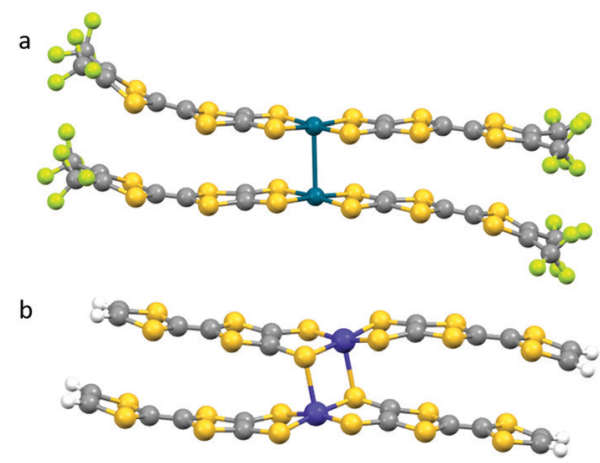

Fig. 7 Details in the crystal structure of (a) $\left[\mathrm{Pd}(\mathrm{hfdt})_{2}\right]$ and (b) $\left[\mathrm{Co}(\mathrm{dt})_{2}\right]$ showing dimers with a metal-metal bonding and with a metal-sulphur bonding, respectively.

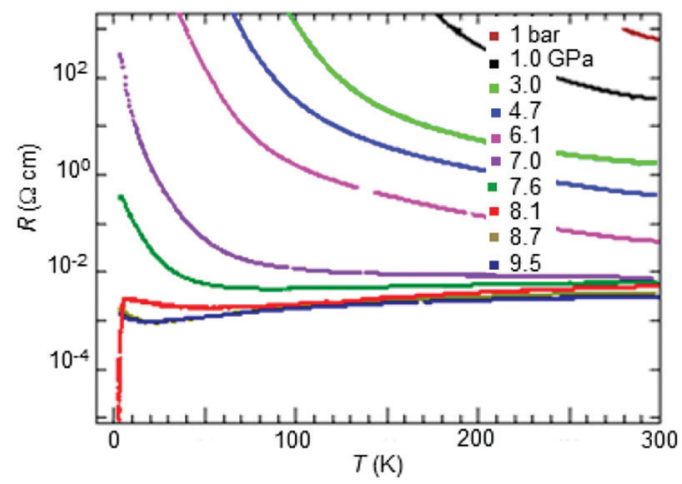

Fig. 8 Temperature dependence of electrical resistivity $(R)$ of $\left[\mathrm{Ni}(\mathrm{hfdt})_{2}\right]$ up to $\sim 10 \mathrm{GPa}$. Adapted with permission from Cui, H., Kobayashi, H., Ishibashi, S., Sasa, M., Iwase, F., Kato, R., \& Kobayashi, A. (2014). A Single-Component Molecular Superconductor. J. Am. Chem. Soc., 136(21), 7619-7622. DOI: 10.1021/ja503690m. Copyright 2014 American Chemical Society.

The $\left[\mathrm{Ni}(\mathrm{ptdt})_{2}\right]$ (ptdt: propylenedithiotetrathiafulvalenedithiolate, Chart 3) presented in single crystal a $\sigma_{\mathrm{RT}}$ of $7 \mathrm{~S} \mathrm{~cm}^{-1}, 55$ and its tight-binding band structure calculation showed the presence of crossing bands, whose influence could only be suppressed by application of high pressures around $19 \mathrm{GPa}$, metallizing this system. ${ }^{56}$ Its gold analogue was also prepared and revealed a crystal structure composed of $2 \mathrm{D}$ layers of the molecules separated by outer rings. The $\left[\mathrm{Au}(\mathrm{ptdt})_{2}\right]$ displayed, on a compressed pellet, a $\sigma_{\mathrm{RT}}$ around $2 \mathrm{~S} \mathrm{~cm}^{-1}$ and a low temperature-independent paramagnetic susceptibility of $1.0 \times$ $10^{-5}$ emu $\mathrm{mol}^{-1}$. $^{-1}$

In the family $\left[\mathrm{M}(\mathrm{dt})_{2}\right]$ (dt: tetrathiafulvalenedithiolate; $\mathrm{M}: \mathrm{Ni}$, Co, Pd and $\mathrm{Zn}$, Chart 3), the $\mathrm{Zn},{ }^{44} \mathrm{Ni}^{20}$ and $\mathrm{Pt}^{58}$ behave as semiconducting systems. The most interesting complex of this family is $\left[\mathrm{Co}(\mathrm{dt})_{2}\right]$, which is a neutral cobalt complex dimerised with metal-sulphur bonds and an intradimer distance of $3.12 \AA$ (Fig. 7b). ${ }^{14,49,59}\left[\mathrm{Co}(\mathrm{dt})_{2}\right]$ displays a $\sigma_{\mathrm{RT}}$ of $19 \mathrm{~S} \mathrm{~cm}^{-1}$ measured in a compressed pellet, and a $\chi_{\mathrm{RT}}$ of $3.5 \times 10^{-4} \mathrm{emu} \mathrm{mol}^{-1}$ that remains practically constant down to $50 \mathrm{~K}$. These results, along with the extended Hückel tight-binding band calculations, suggest that $\left[\mathrm{Co}(\mathrm{dt})_{2}\right]$ is a dimerised SCMM. ${ }^{14,49,59}$

The incorporation of $\pi$-conjugated substituents on the TTF moiety could be a strategy to retain the conducting properties of the final complexes. [Ni(btdt $\left.)_{2}\right]$ (btdt: benzotetrathiafulvalenedithiolate, Chart 3) is an example of a bis(1,2-dithiolene) complex with a phenyl fused with the TTF moiety resulting in a highly conjugated and conducting complex. ${ }^{60}$ Its crystal structure is composed of planar molecules connected by a 3D network of $S \cdots S$ contacts (less than $3.60 \AA$ ) and $\pi \cdots \pi$ interactions. This complex behaves as a narrow-gap semiconductor with a $\sigma_{\mathrm{RT}}$ measured in compressed pellets of $10 \mathrm{~S} \mathrm{~cm}^{-1} \cdot{ }^{60}$

Other examples of ligands with extended TTF moieties incorporating $\pi$-conjugated substituents are $\alpha$-tdt $(\alpha$-tdt: thiophenetetrathiafulvalenedithiolate), and the non-aromatic relative dtdt (dtdt: dihydrothiophenetetrathiafulvalenedithiolate) (Chart 3). Once again, the purpose was to increase the $\pi$-delocalisation and, with the additional sulphur atoms, favour the intermolecular interaction in the outer rings. ${ }^{61}$ The neutral complexes obtained by 


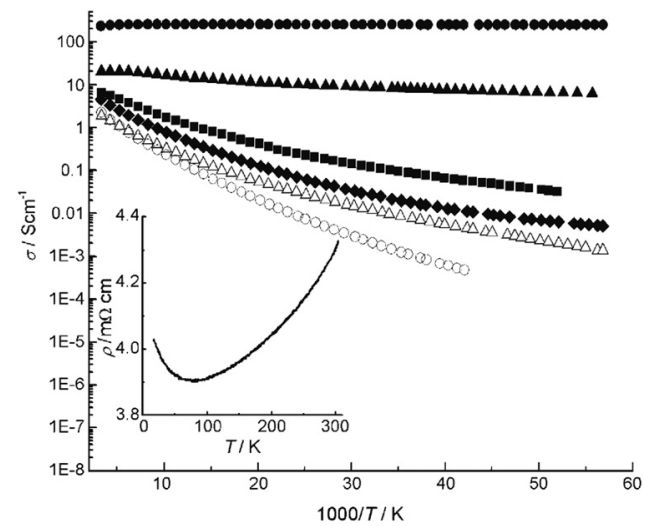

Fig. 9 Temperature dependence of electrical conductivity $(\sigma)$ measured on polycrystalline samples of [Au(dtdt) $\left.)_{2}\right](\boldsymbol{)}),\left[\mathrm{Au}(\alpha-\mathrm{tdt})_{2}\right](\mathbf{\square})$, [Ni(dtdt) $\left.{ }_{2}\right]($ oxidation by air exposure, $\mathrm{O}$ : iodine oxidation) and $\left[\mathrm{Ni}(\alpha-\mathrm{tdt})_{2}\right](\boldsymbol{\Lambda}$ : oxidation by air exposure, $\triangle$ : iodine oxidation). Inset: Temperature dependence of electrical resistivity $(\rho)$ of $\left[\mathrm{Ni}(\mathrm{dtdt})_{2}\right]$, obtained by oxidation by air exposure. Reprinted with permission from ref. 61. Copyright (C)2007 WILEY-VCH Verlag GmbH \& Co. KGaA, Weinheim.

iodine oxidation, showed conductivities of 2 and $2.5 \mathrm{~S} \mathrm{~cm}^{-1}$ for $\left[\mathrm{Ni}(\alpha-\mathrm{tdt})_{2}\right]$ and $\left[\mathrm{Ni}(\mathrm{dtdt})_{2}\right]$, respectively, and slightly higher for $\left[\mathrm{Au}(\alpha-\mathrm{tdt})_{2}\right]$ and $\left[\mathrm{Au}(\mathrm{dtdt})_{2}\right]$, of 5 and $8 \mathrm{~S} \mathrm{~cm}^{-1}$, respectively. However, slow oxidation through air exposure led to higher crystalline neutral nickel complexes that display higher $\sigma_{\mathrm{RT}}$, of 200 and $24 \mathrm{~S} \mathrm{~cm}^{-1}$ for $\left[\mathrm{Ni}(\mathrm{dtdt})_{2}\right]$ and $\left[\mathrm{Ni}(\alpha-\mathrm{tdt})_{2}\right]$, respectively. These samples present behaviours typical of metals with conductivity increasing upon cooling (Fig. 9). ${ }^{61}$ Oddly enough, the higher $\sigma_{\mathrm{RT}}$ values were obtained by the non-aromatic analogues. The differences in the values of the conductivity found upon using different oxidation methodologies may suggest different degrees of crystallinity or the formation of different phases. All compounds display rather large magnetic susceptibilities, corresponding to effective magnetic moments in the range 1-3 $\mu_{\mathrm{B}}$, which may be indicative of high spin states. It is worthwhile to mention that $\left[\mathrm{Ni}(\mathrm{dtdt})_{2}\right]$ films showed related properties to commercial carbon-based conductive inks. ${ }^{62}$ The copper analogues $\left[\mathrm{Cu}(\mathrm{dtdt})_{2}\right]$ and $\left[\mathrm{Cu}(\alpha \text {-tdt })_{2}\right]$ were also prepared showing electric conductivities, ranging between $0.10-0.40 \mathrm{~S} \mathrm{~cm}^{-1}$, however with a semiconducting behaviour. ${ }^{63}$

The methyl substituted $\left[\mathrm{M}(\alpha-\mathrm{tdt})_{2}\right]$ analogues, $\left[\mathrm{Au}(\alpha-\mathrm{mtdt})_{2}\right]$ and $\left[\mathrm{Ni}(\alpha-\mathrm{mtdt})_{2}\right]$ were also prepared and studied. ${ }^{26}$ The electric transport properties, measured in compressed pellets, showed for the nickel complex a $\sigma_{\mathrm{RT}}$ of $3.2 / 4.2 \mathrm{~S} \mathrm{~cm}^{-1}$ (obtained by air exposure/iodine oxidation) and for the gold complex a $\sigma_{\mathrm{RT}}$ of $0.32 \mathrm{~S} \mathrm{~cm}^{-1}$. The lower $\sigma_{\mathrm{RT}}$ observed when compared with $\left[\mathrm{M}(\alpha-\mathrm{tdt})_{2}\right]$, is probably due to the methyl group that prevents intermolecular interactions. ${ }^{26}$

In the $\left[\mathrm{M}(\mathrm{tmstfdt})_{2}\right]$ family (tmstfdt: trimethylenediselenadithiafulvalenedithiolate; $\mathrm{M}$ : $\mathrm{Au}$ and $\mathrm{Ni}$, Chart 3), ${ }^{64}$ some sulphur atoms are replaced by selenium atoms in the tmdt ligand, which could improve some intermolecular interaction since the selenium is bulkier. The structure of these complexes is isostructural with $\left[\mathrm{M}(\mathrm{tmdt})_{2}\right]$ complexes $(\mathrm{M}: \mathrm{Ni}, \mathrm{Au}, \mathrm{Cu}, \mathrm{Pd}$ and $\mathrm{Pt}$ ). Their crystal structures form a $3 \mathrm{D}$ network of short intermolecular $\mathrm{S}(\mathrm{Se}) \cdots \mathrm{S}(\mathrm{Se})$ contacts (the shortest $\mathrm{S} \cdots \mathrm{S}$ contact value is $3.49 \AA$ ). Measurements of the electrical conductivity, in compressed pellets, revealed values of 100 and $11 \mathrm{~S} \mathrm{~cm}^{-1}$ for the $\mathrm{Ni}$ and $\mathrm{Au}$ complexes, respectively. ${ }^{64}$ The $\left[\mathrm{Ni}(\mathrm{tmstfdt})_{2}\right]$ displays metallic properties down to $50 \mathrm{~K}$ and retains high conductivity at very low temperature $\left(\sigma_{4.2 \mathrm{~K}} \approx \sigma_{\mathrm{RT}}\right)$ while the gold complex shows a semiconducting behaviour, nevertheless showing above $100 \mathrm{~K}$ a constant magnetic susceptibility reminiscent of a metallic system. It seems that the substitution for Se atoms did not benefit the conducting properties, even when they seem to enhance the intermolecular interactions, which shows that this substitution with bulkier Se atoms is not so straightforward. ${ }^{64}$

$\mathrm{Au}\left[(\text { pesdt })_{2}\right]$ (pesdt: pyridylethylsulfanyltetrathiafulvalenedithiolene, Chart 3$)^{65}$ and $\left[\mathrm{M}(\mathrm{pztdt})_{2}\right]$ (pztdt: pyrazinetetrathiafulvalenedithiolate, $\mathrm{M}$ : Ni, Pd and Pt, Chart 3) complexes were also reported, but with no relevant features. ${ }^{66}$

Several years of research of these extended TTF 1,2-dithiolene complexes, provided important information about how to accomplish a metallic state on a neutral species. Additionally, they show that the end capping groups seem to be less important to the solid-state properties. For instance, there is no significant differences on conductivities of $\left[\mathrm{Ni}(\mathrm{tmdt})_{2}\right]$ and $\left[\mathrm{Ni}(\mathrm{dmdt})_{2}\right]$, which have a fused ring and methyl groups, respectively. The electronic density is essentially on the TTF unit and not on its substituents, and neither in the metal. This suggests that the TTF core is the main responsible for the solid-state properties. The substituents usually do not contribute for the overlapping and bulkier groups can even prevent it. Furthermore, metals seem to retain the magnetic properties, so it is possible to have conducting complexes with special magnetic behaviours.

\section{BTM Complexes with thiophene/selenophene rings}

Contemporary to $\left[\mathrm{Ni}(\mathrm{tmdt})_{2}\right]$ are the smaller gold bis(1,2-dithiolene) complexes with thiophene rings. The most promising one was $\left[\mathrm{Au}(\alpha \text {-tpdt })_{2}\right]$, the first SCMM without a fused TTF moiety incorporated in the molecular structure (Chart 3). This gold complex shows a relatively high $\sigma_{\mathrm{RT}}$ of $7 \mathrm{~S} \mathrm{~cm}^{-1}$ for a measurement in a polycrystalline pressed pellet (Fig. 10). ${ }^{12}$ Although the conductivity

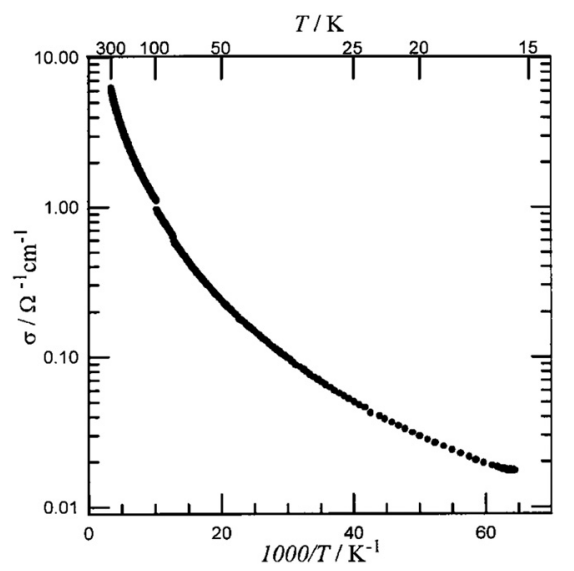

Fig. 10 Temperature dependence of the electric conductivity measured in a polycrystalline sample of $\left[\mathrm{Au}(\alpha-\mathrm{tpdt})_{2}\right]$. Reprinted with permission from ref. 12. (C)2001 Wiley-VCH Verlag GmbH, Weinheim, Fed. Rep. of Germany. 
decreases with temperature, the thermopower measurements and the magnetic properties, which revealed a Pauli-like paramagnetism, show a behaviour consistent with a metallic system. Furthermore, thin polycarbonate films metalized with $\left[\mathrm{Au}(\alpha \text {-tpdt })_{2}\right]$ were found to be a strain-resistive material with advanced elastic properties and clearly show the metallic character of this gold complex. ${ }^{67}$

Analogues of this first small SCMM gold complex [Au(tpdt $\left.)_{2}\right]$ (tpdt: 3,4-thiophenedithiolate) and [Au(dtpdt) $\left.{ }_{2}\right]$ (dtpdt: 2,3-dihydro5,6-thiophenedithiolate) showed different conducting behaviours (Chart 3). ${ }^{12}$ Their electric transport properties were also measured in compressed pellets and showed a $\sigma_{\mathrm{RT}}$ of $10^{-9} \mathrm{~S} \mathrm{~cm}^{-1}$ and $3.3 \times$ $10^{-6} \mathrm{~S} \mathrm{~cm}^{-1}$, respectively, revealing a semiconducting behaviour. These neutral salts are insoluble in common organic solvents, preventing all attempts to obtain single crystals. One approach to overcome this drawback is to incorporate solubilizing alkyl chains in the molecular framework. Following this strategy different families of derivatives with a methyl, ${ }^{68}$ a tert-butyl ${ }^{69}$ and a diisopropyl, ${ }^{69}$ in the outer thiophene ring were prepared (Chart 3). The transport properties of $\left[\mathrm{Au}(\alpha-\mathrm{mtpdt})_{2}\right]$ and $\left[\mathrm{Ni}(\alpha-\mathrm{mtpdt})_{2}\right]$ ( $\alpha$-mtpdt: 5-methylthiophene-2,3-dithiolate, Chart 3), performed in compressed pellets, revealed in both cases a semiconducting behaviour, with a $\sigma_{\mathrm{RT}}$ of $5.2 \times 10^{-7} \mathrm{~S} \mathrm{~cm}^{-1}$ and of $8.7 \times 10^{-5} \mathrm{~S} \mathrm{~cm}^{-1}$ for the nickel and gold complexes, respectively. The crystal structure of $\left[\mathrm{Ni}(\alpha-\mathrm{mtpdt})_{2}\right]$ shows a poor intermolecular overlapping, prevented by the methyl groups. ${ }^{68}\left[\mathrm{M}(\alpha-t b \text {-tpdt })_{2}\right]$ ( $\alpha$-tb-tpdt: 5 -(tert-butyl)thieno[2,3- $d][1,3]$ dithiolate $)$ and $\quad\left[\mathrm{M}(\alpha \text {-dp-tpdt })_{2}\right]$ ( $\alpha$-dp-tpdt: 5,6-diisopropylthieno[2,3- $d][1,3]$ dithiolate) (M: $\mathrm{Au}$ and $\mathrm{Ni}$, Chart 3 ) displayed electric conductivity values ranging between $10^{-8}$ and $5 \times 10^{-5} \mathrm{~S} \mathrm{~cm}^{-1}$, measured in single crystal. In these complexes the bulky substituent groups impede both successful overlapping and intermolecular interactions, thus presenting insulating behaviours, due to large HOMO-LUMO gaps. Additionally, in neutral gold complexes the presence of one electron in the HOMO leads to a half-filled band and the system behaves as a Mott insulator. ${ }^{69}$ The ligand bond lengths asymmetry present on $\left[\mathrm{Au}(\alpha \text {-tb-tpdt })_{2}\right]$ suggests the localisation of the unpaired electron in one ligand, while in $\left[\mathrm{Au}(\alpha-\mathrm{dp}-\mathrm{tpdt})_{2}\right]$ the bond lengths are fully symmetric. Consequently, $[\mathrm{Au}(\alpha-\mathrm{tb}-$ tpdt $)_{2}$ ] presents a dimerised arrangement in the solid state, resulting in a diamagnetic behaviour, while $\left[\mathrm{Au}(\alpha \text {-dp-tpdt })_{2}\right]$ organized in regular stacks, is subject of strong antiferromagnetic interactions and an antiferromagnetic transition around $25 \mathrm{~K}{ }^{69}$

Other analogues of $\left[\mathrm{Au}(\alpha \text {-tpdt })_{2}\right]$ disubstituted with methyl and ethyl groups revealed a low $\sigma_{\mathrm{RT}}$ of $0.003 \mathrm{~S} \mathrm{~cm}^{-1}$ for $[\mathrm{Au}(\alpha-$ EtMetpdt $\left.)_{2}\right]$ ( $\alpha$-EtMetpdt: 4-ethyl-5-methyl-thiophene-2,3-dithiolate, Chart 3), measured in single crystal, and $0.01 \mathrm{~S} \mathrm{~cm}^{-1}$ for $[\mathrm{Au}(\alpha-$ $\mathrm{Me}_{2}$ tpdt $)_{2}$ ] ( $\alpha$-Me $\mathrm{Me}_{2}$ dt: 4,5-dimethyl-thiophene-2,3-dithiolate, Chart 3), measured in compressed pellet. ${ }^{70}$ The lack of crystal data prevents a deep understanding of the solid-state properties of this thiophenic family. However, the functionalization of small BTM by introducing bulky substituents, needs to be balanced since small changes in the molecular structure can alter drastically the material transport properties.

In the neutral complex [Au(dspdt) $)_{2}$ (dspdt: 2,3-dihydro5,6-selenophenedithiolate, Chart 3), a selenium analogue of $\left[\mathrm{Au}(\mathrm{dtpdt})_{2}\right]$, the crystal structure is composed of an unique
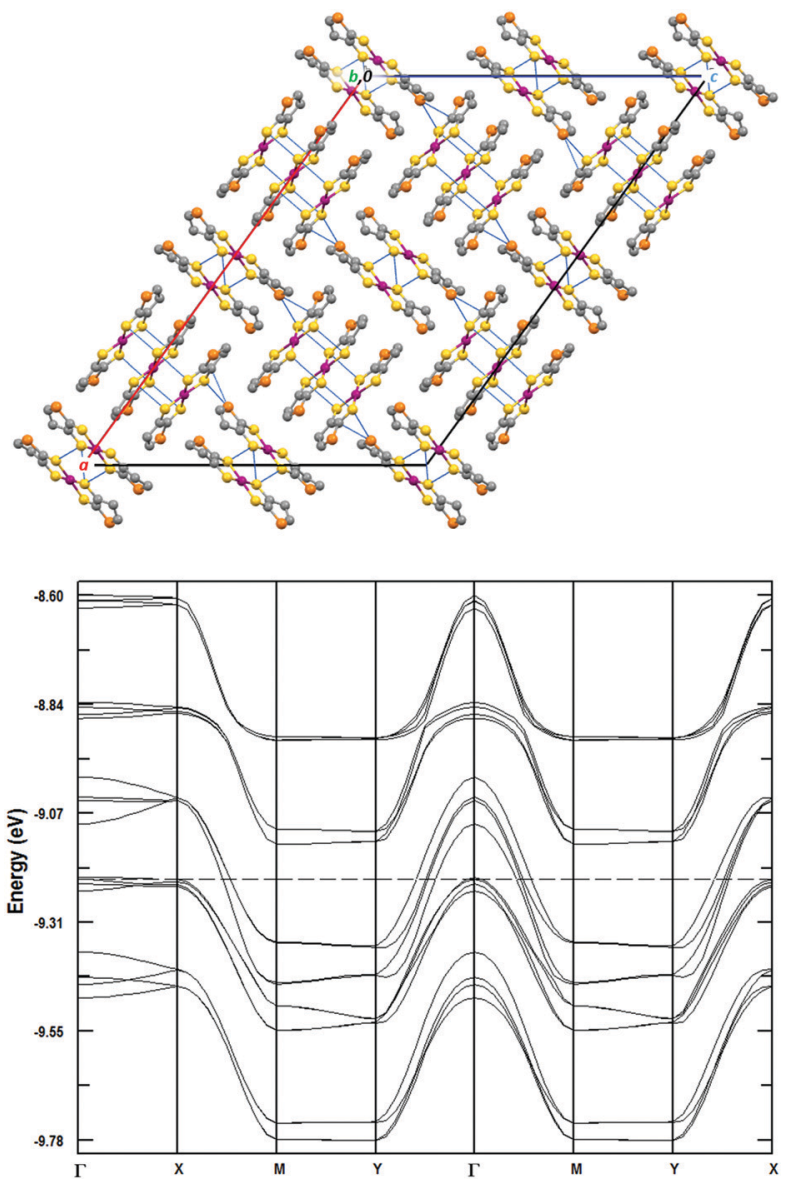

Fig. 11 Top: Crystal structure of [Au(dspdt) $)_{2}$ ] viewed along $b$ axis (only one trans configuration for the disordered Se atoms was considered). The thin blue lines denote short contacts. Bottom: Calculated band structure, 20 SOMO bands near the Fermi level (dashed line). The. $\Gamma, X, Y$ and $M$ refer to the $(0,0,0),(1 / 2,0,0),(0,1 / 2,0),(1 / 2,1 / 2,0)$, points in $k$-space, respectively. Reproduced from ref. 71 with permission from The Royal Society of Chemistry.

pattern of interacting columns of dimers and columns of trimers, running along $b$ (Fig. 11). ${ }^{71}\left[\mathrm{Au}(\mathrm{dspdt})_{2}\right]$ revealed a semiconducting behaviour measured in poor quality small single crystals and a $\sigma_{\mathrm{RT}}$ of $0.1 \mathrm{~S} \mathrm{~cm}^{-1}$ with an activation energy of $0.095 \mathrm{eV}$. However, theoretical calculations evidence a band structure with parallel bands considerably split and partially overlapping, with the four central bands crossing the Fermi level at different wave vectors, suggesting a metallic behaviour. Nevertheless, this behaviour is not observed experimentally because the tight-binding extended Hückel model does not consider electron correlation effects. ${ }^{71}$

\section{BTM complexes with thiazole rings}

Anionic bis(1,2-dithiolene) TM complexes with thiazole rings were first prepared by M. Arca et al. ${ }^{72}$ The first neutral complex prepared was $\left[\mathrm{Au}(\text { Et-thiazdt })_{2}\right]$ (Et-thiazdt: $\mathrm{N}$-ethyl-1,3-thiazoline-2thione-4,5-dithiolate, Chart 3). ${ }^{21}$ The crystalline structure of this complex shows uniform stacks with a lateral slip between neighbouring molecules and several $\mathrm{S} \cdots \mathrm{S}$ contacts $(3.419 \AA$ $3.841 \AA$ ). Single crystals at ambient pressure presented a $\sigma_{\mathrm{RT}}$ of 

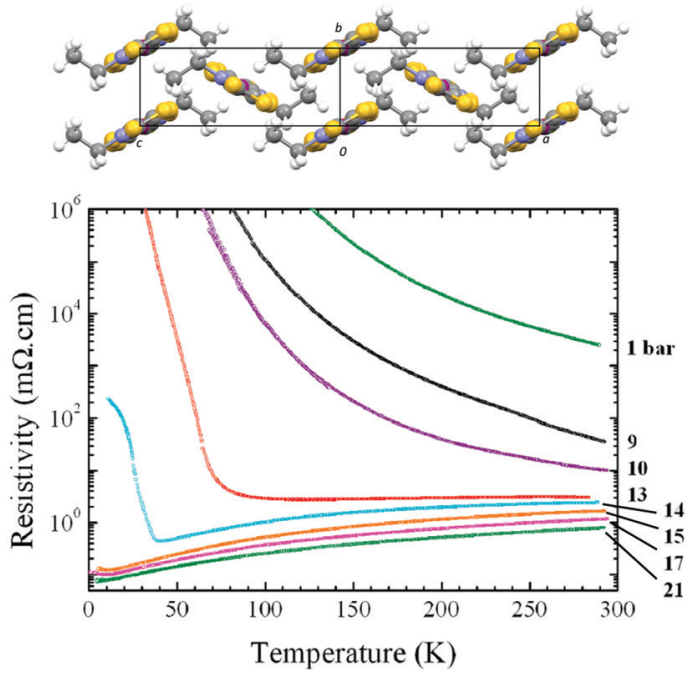

Fig. 12 Top: Detail showing the molecular stacking along $b$, in the crystal structure of $\left[\mathrm{Au}(\mathrm{Et}-\mathrm{thiazdt})_{2}\right]$. Bottom: Temperature dependence of the resistivity of $\left[\mathrm{Au}(\mathrm{Et}-\mathrm{thiazdt})_{2}\right]$ at different applied pressures. Adapted with permission from Tenn, N., Bellec, N., Jeannin, O., Piekara-Sady, L., AubanSenzier, P., Íniguez, J., Canadell, E., \& Lorcy, D. (2009). A Single-Component Molecular Metal Based on a Thiazole Dithiolate Gold Complex. J. Am. Chem. Soc., 131(46), 16961-16967. DOI: 10.1021/ja907426s. Copyright 2009 American Chemical Society.

$0.33 \mathrm{~S} \mathrm{~cm}^{-1}$ with a semiconducting behaviour. However, its conductivity is highly dependent on the pressure (Fig. 12), with a metal to insulator transition being observed at $13 \mathrm{kbar}$ below $70 \mathrm{~K}$. This transition is suppressed by applying pressure, and the compound reaches a $\sigma_{\mathrm{RT}}$ of $1000 \mathrm{~S} \mathrm{~cm}^{-1}$ at $21 \mathrm{kbar}^{21}$ Despite the semiconducting behaviour at ambient pressure the magnetic susceptibility measurements revealed a Pauli-like behaviour, typical of a metallic system in agreement with spin-charge separation in 1D correlated systems. ${ }^{21}$

Systematic changes have been made in the chemical molecular structure of $\left[\mathrm{Au}(\mathrm{Et} \text {-thiazdt })_{2}\right]$ focusing on the substituents (Me, Et, EtOH, $\mathrm{NMe}_{2}, \mathrm{iPr}, c \mathrm{Pr}$ or $n \mathrm{Pr}$ ), the central TM (Au, Ni or $\mathrm{Pt}$ ), the chalcogenones ( $\mathrm{S}$ or $\mathrm{O}$ ), and coordination atoms ( $\mathrm{S}$ or Se) (Chart 3).

$\left[\mathrm{Au}(\text { Et-thiazodt })_{2}\right]\left(\right.$ or $\operatorname{AuS}_{4}(=\mathrm{O})_{2}$; thiazods: 1,3-thiazoline-2one-4,5-dithiolate), $\left[\mathrm{Au}(\text { Et-thiazds) })_{2}\right]$ (or $\operatorname{AuSe}_{4}(=\mathrm{S})_{2}$; thiazds: 1,3-thiazoline-2-thione-4,5-diselenolate) and [Au(Et-thiazods) $\left.)_{2}\right]$ (or $\mathrm{AuSe}_{4}(=\mathrm{O})_{2}$; thiazods: 1,3-thiazoline-2-one-4,5-diselenolate) are three derivatives of $\left[\mathrm{Au}(\mathrm{Et} \text {-thiazdt })_{2}\right]$ with different chalcogen atoms (Chart 3). The four neutral complexes are isostructural. The crystal structures are composed of uniform stacks connected by lateral $\mathrm{S}(\mathrm{Se}) \cdots \mathrm{S}(\mathrm{Se})$ interactions. At ambient pressure, the resistivity measurements revealed a $\sigma_{\mathrm{RT}}$ of $0.33 \times 10^{-2}, 0.18$ and $40 \mathrm{~S} \mathrm{~cm}^{-1}$ for $\left[\operatorname{AuS}_{4}(=\mathrm{O})_{2}\right],\left[\operatorname{AuSe}_{4}(=\mathrm{O})_{2}\right]$ and $\left[\operatorname{AuSe}_{4}(=\mathrm{S})_{2}\right]$, respectively (Fig. 13). Replacing the coordinating sulphur with selenium atoms increased the $\sigma_{\mathrm{RT}}$ by two orders of magnitude. This substitution mainly affects the axis associated with lateral interactions, revealing the anisotropy of the system. ${ }^{73}$ However, the replacement with oxygen decreased the $\sigma_{\mathrm{RT}}$ by two orders of magnitude. In the case of $\left[\operatorname{AuSe}_{4}(=\mathrm{O})_{2}\right]$, where both changes occur simultaneously their effects seem to cancel each other. ${ }^{73}$
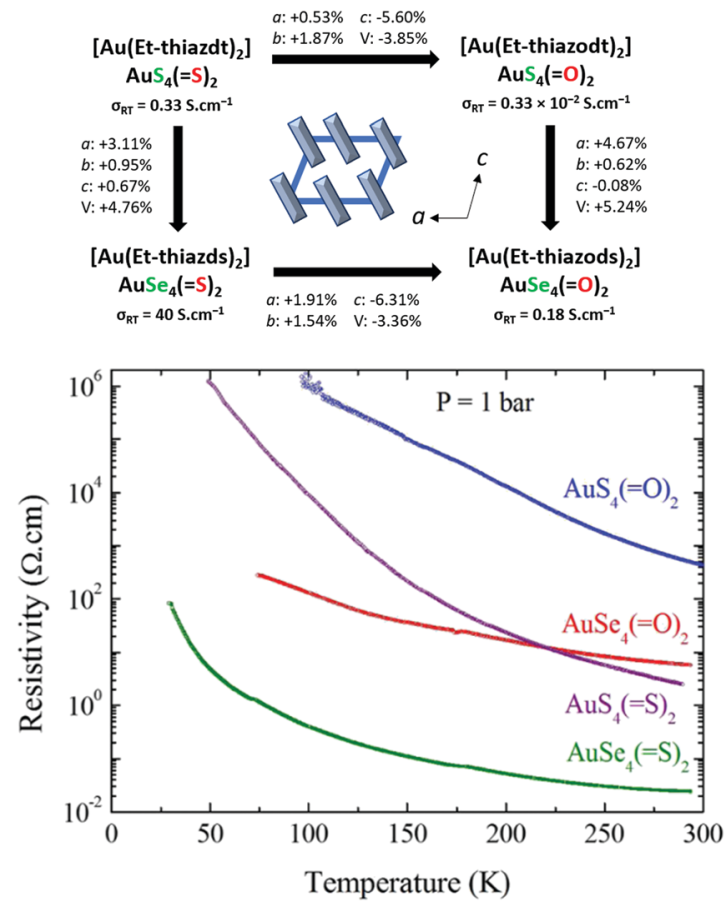

Fig. 13 Top: Evolution of the unit cell parameters within the series. Bottom: Temperature dependence of the resistivity of the four complexes at ambient pressure measured in single crystals. Adapted with permission from Yzambart, G., Bellec, N., Nasser, G., Jeannin, O., Roisnel, T., Fourmigué, M., Auban-Senzier, P., İ̃̃iguez, J., Canadell, E, \& Lorcy, D. (2012). Anisotropic Chemical Pressure Effects in Single-Component Molecular Metals Based on Radical Dithiolene and Diselenolene Gold Complexes. J. Am. Chem. Soc., 134(41), 17138-17148. DOI: 10.1021/ja3065649. Copyright 2012 American Chemical Society.

$\left[\operatorname{AuSe}_{4}(=\mathrm{S})_{2}\right]$ displays higher $\sigma_{\mathrm{RT}}$ under pressure, reaching $2600 \mathrm{~S} \mathrm{~cm}^{-1}$ at $12.5 \mathrm{kbar}$. A typical semiconducting behaviour is observed until 4 kbar with a metallic behaviour seen above 6 kbar (Fig. 14), indicating a higher sensitivity of the structure to pressure at variance with $\left[\operatorname{AuS}_{4}(=\mathrm{S})_{2}\right]$, where this change occurs at higher pressures (13-14 kbar). Here, the Se substitution for $\mathrm{S}$ efficiently reduces the gap. ${ }^{73}$

$\left[\mathrm{Ni}(\text { Et-thiazdt })_{2}\right]^{74}$ is not isostructural with its gold analogue. The crystal structure is still composed of regular stacks of the neutral molecules, but with a different overlapping pattern, showing a large molecular longitudinal slide. Due to a lack of an electron, the crystal structure, in this case ends up being controlled by intermolecular interactions. ${ }^{74}$ Despite its closedshell character, [Ni(Et-thiazdt $)_{2}$ ] displays a $\sigma_{\mathrm{RT}}$ of approximately $0.014 \mathrm{~S} \mathrm{~cm}^{-1}$, revealing a semiconducting behaviour. ${ }^{74}$ Afterwards, a new [Ni(Et-thiazdt $)_{2}$ ] phase was found and although it revealed again a semiconducting behaviour with a low $\sigma_{\mathrm{RT}}$ of $4.3 \times 10^{-4} \mathrm{~S} \mathrm{~cm}^{-1}$ it also became metallic for a large range of temperatures when under a pressure of $6.9 \mathrm{GPa}^{75}$

A series of $\left[\mathrm{Au}(\mathrm{R} \text {-thiazdt })_{2}\right.$ ] were also prepared with different substituents, such as R: EtOH, ${ }^{76}{ }^{\mathrm{i}} \mathrm{Pr},{ }^{77}{ }^{c} \mathrm{Pr},{ }^{78} \mathrm{Pr},{ }^{78} \mathrm{NMe}_{2}{ }^{78}$ and Me. ${ }^{79}\left[\mathrm{Au}(\mathrm{EtOH} \text {-thiazdt })_{2}\right]$ revealed a semiconducting behaviour with a $\sigma_{\mathrm{RT}}$ of $0.05-0.07 \mathrm{~S} \mathrm{~cm}^{-1} \cdot{ }^{76}$ In turn, the analogue with ${ }^{\mathrm{i}} \mathrm{Pr}$ bulky groups adopted a crisscross overlap structure, which permits strong interactions. This complex exhibits at ambient 


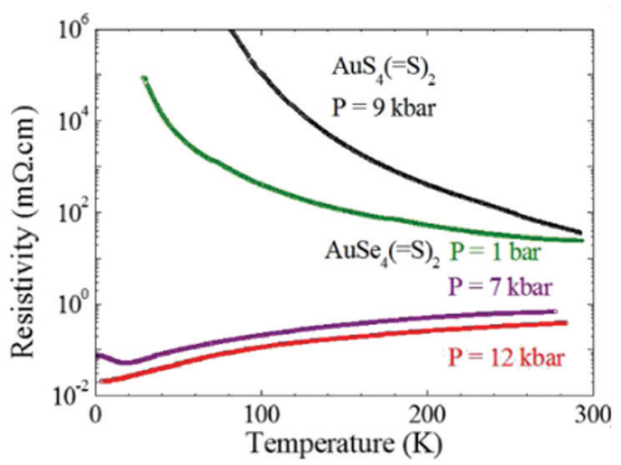

Fig. 14 Temperature dependence of the resistivity of $\left[\mathrm{AuSe}_{4}(=\mathrm{S})_{2}\right]$ at different pressures. Adapted with permission from Yzambart, G., Bellec, N., Nasser, G., Jeannin, O., Roisnel, T., Fourmigué, M., Auban-Senzier, P., Iñiguez, J., Canadell, E, \& Lorcy, D. (2012). Anisotropic Chemical Pressure Effects in Single-Component Molecular Metals Based on Radical Dithiolene and Diselenolene Gold Complexes. J. Am. Chem. Soc., 134(41), 17138-17148. DOI: 10.1021/ja3065649. Copyright 2012 American Chemical Society.

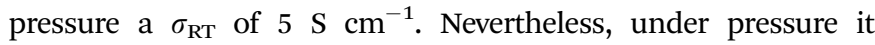
maintains its non-metallic behaviour due to the [Au(iPr-thiazdt) $)_{2}$ ] direct band gap resulting from an avoided band crossing. ${ }^{77}$ Although of similar structure with $\left[\mathrm{Au}\left(\mathrm{iPr}\right.\right.$-thiazdt) $\left.{ }_{2}\right]$, the $\mathrm{NMe}_{2}$ substituents show different solid-state properties, with $\left[\mathrm{Au}\left(\mathrm{NMe}_{2}-\right.\right.$ thiazdt) ${ }_{2}$ ] achieving a $\sigma_{\mathrm{RT}}$ of only $0.045 \mathrm{~S} \mathrm{~cm}^{-1} .^{78}$

In this thiazole rings family the $\left[\mathrm{Au}(\mathrm{Me}-\mathrm{thiazdt})_{2}\right]$ complex was the first SCMM at ambient pressure. ${ }^{79}$ With a crystal structure similar to $\left[\mathrm{Au}(\text { Et-thiazdt })_{2}\right]$, this complex presents a 2D character with several significantly short S $\cdots S$ contacts (3.306-3.727 ̊). These contacts are not only shorter than the ones in $\left[\mathrm{Au}(\text { Et-thiazdt })_{2}\right]$, but there is also an extra $\mathrm{S} \cdots \mathrm{S}$ intermolecular contact $(3.567 \AA)$ between the exocyclic [Au(Methiazdt $)_{2}$ ] sulphur atoms (Fig. 15 inset). ${ }^{79}$ The metallic behaviour of this system is in agreement with the observed $\sigma_{\mathrm{RT}}$ of $750 \mathrm{~S} \mathrm{~cm}^{-1}$ (Fig. 15), measured in single crystal, and a Pauli-like paramagnetic

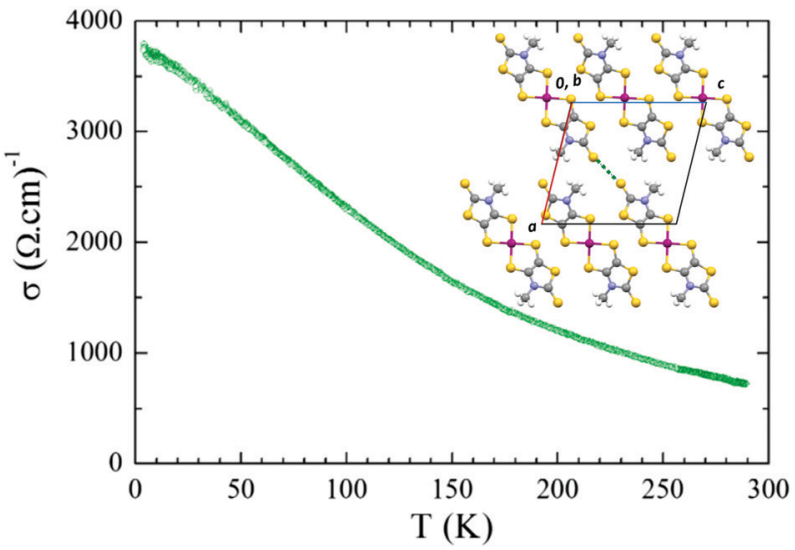

Fig. 15 Temperature dependence of the conductivity of [Au(Me-thiazdt) $)_{2}$; Inset: Crystal structure of [Au(Me-thiazdt) $)_{2}$ ] viewed along $b$. Adapted with permission from Le Gal, Y., Roisnel, T., Auban-Senzier, P., Bellec, N., İñiguez, J., Canadell, E., \& Lorcy, D. (2018). Stable Metallic State of a Neutral-Radical Single-Component Conductor at Ambient Pressure. J. Am. Chem. Soc., 140(22), 6998-7004. DOI: 10.1021/jacs.8b03714. Copyright 2018 American Chemical Society. susceptibility of $3.2 \times 10^{-4}$ emu $\mathrm{mol}^{-1}$. $^{79}$ The analogue [Ni(Methiazdt $)_{2}$ ] presented semiconducting behaviour with a $\sigma_{\mathrm{RT}}$ of $0.05 \mathrm{~S} \mathrm{~cm}^{-1}$, higher than the observed in the other Ni complexes of this family. ${ }^{75}$ Under pressure the $\sigma_{\mathrm{RT}}$ was enhanced to $166 \mathrm{~S} \mathrm{~cm}^{-1}$ at $19 \mathrm{GPa}$, however its semiconducting behaviour remains. ${ }^{75}$

In both $\left[\mathrm{Pt}(\mathrm{R} \text {-thiazdt })_{2}\right]$ (R: Me and $\left.\mathrm{Et}\right)^{75}$ a semiconducting behaviour was observed. At $12 \mathrm{GPa}$, the ethyl analogue still behaves as a semiconductor.

Another family with thiazole rings worth mentioning is the series $\left[\mathrm{M}(\mathrm{RS} \text {-tzdt })_{2}\right]$ (RS-tzdt: 2-alkyl-thio-1,3-thiazole-4,5dithiolate; $\mathrm{M}: \mathrm{Au}^{80}$ and $\mathrm{Ni} ;{ }^{81} \mathrm{R}$ : Et, Me and $\mathrm{EtOH},{ }^{82}$ Chart 3). Regarding $\left[\mathrm{Au}(\mathrm{RS}-\mathrm{tzdt})_{2}\right]$ with $\mathrm{Me}$ and Et substituents both revealed a semiconducting behaviour with a $\sigma_{\mathrm{RT}}$ of $8 \times 10^{-3} \mathrm{~S} \mathrm{~cm}^{-1}\left(E_{\mathrm{a}}=\right.$ $0.13 \mathrm{eV})$ and $3.3 \times 10^{-4} \mathrm{~S} \mathrm{~cm}^{-1}\left(E_{\mathrm{a}}=0.12 \mathrm{eV}\right)$, respectively. ${ }^{80} \mathrm{In}$ [Ni(RS-tzdt $)_{2}$ ] (R: Me, Et) a semiconducting behaviour was observed, with $\sigma_{\mathrm{RT}}$ ranging between $5 \times 10^{-7}-5 \times 10^{-6} \mathrm{~S} \mathrm{~cm}^{-1}$ and between $10^{-4}-10^{-5} \mathrm{~S} \mathrm{~cm}^{-1}$ for the Me and Et substituted complexes, respectively. ${ }^{81}$

The gold and nickel complexes of $\left[\mathrm{M}(\text { EtOHS-tzdt })_{2}\right]$ were found to be isostructural presenting uniform stacks and strong $\mathrm{O}-\mathrm{H} \cdots \mathrm{N}$ hydrogen bonds $(1.968 \AA$ for the Ni complex and $2.065 \AA$ for the Au complex) between molecules in neighbouring stacks. The hydrogen bonds control the overall crystal structures regardless of their different open/closed shell nature. ${ }^{82}$ The nickel complex behaves as a band semiconductor with a weak $\sigma_{\mathrm{RT}}\left(1.6 \times 10^{-5} \mathrm{~S} \mathrm{~cm}^{-1}\right)$, while the gold complex is described as a Mott insulator with a $\sigma_{\mathrm{RT}}$ three orders of magnitude higher $\left(0.06 \mathrm{~S} \mathrm{~cm}^{-1}\right)$.

The last member of this $\left[\mathrm{M}(\mathrm{RS} \text {-tzdt })_{2}\right]$ family is $\left[\mathrm{Au}(\mathrm{I} \text {-tzdt })_{2}\right]$ (I-tzdt: 2-iodo-1,3-thiazole-4,5-dithiolate, Chart 3), that as a compressed pellet, displays a $\sigma_{\mathrm{RT}}$ of $4-5 \times 10^{-3} \mathrm{~S} \mathrm{~cm}^{-1} \cdot{ }^{83}$

In this thiazole family, and as in the thiophene/selenophene the molecular system is very sensitive to the introduction of bulkier substituent groups, precluding in some cases the molecular interactions, affecting the transport properties. The molecular interactions in these cases may also be affected by open/close shell nature of the central TM, since the number of electrons in the molecule may condition the molecular overlapping modes. In addition, the substitution of the coordinating $\mathrm{S}$ for Se revealed to be efficient in the enlargement of the system dimensionality.

\section{BTM complexes with sulphur/selenium heterocycles}

The anionic $\left[\mathrm{M}(\mathrm{dmit})_{2}\right]$ (Chart 3) complexes were extensively used to prepare charge transfer salts that present very promising conducting properties. The neutral $\left[\mathrm{Ni}(\mathrm{dmit})_{2}\right]$ complex was also obtained, presenting a crystal structure composed of uniform stacks of molecules with interstack short S $\cdots$ S contacts $(3.45 \AA$ $3.58 \AA$ ).${ }^{84}$ The resistivity measurements in single crystal show a small $\sigma_{\mathrm{RT}}$ of $3.5 \times 10^{-3} \mathrm{~S} \mathrm{~cm}^{-1} \cdot{ }^{84}$ Almost 30 years later, the pressure dependence was studied revealing a change in the conducting properties, going from semiconductor at low pressure to a metallic state in a wide range of temperatures for pressures above $15.9 \mathrm{GPa}$, reaching a $\sigma_{\mathrm{RT}}$ of $140 \mathrm{~S} \mathrm{~cm}^{-1}$ at $25.5 \mathrm{GPa}^{85}$ The band calculations show the closure of the semiconducting gap and the appearance of $2 \mathrm{D}$ and 3D Fermi 
surfaces under high pressure, which is consistent with the metallic behaviour observed experimentally. ${ }^{85}$ The gold complex, [Au(dmit) 2 ], revealed a semiconducting behaviour with a $\sigma_{\mathrm{RT}}$ of $5.0 \times 10^{-4} \mathrm{~S} \mathrm{~cm}^{-1}$ and an $E_{\mathrm{a}}$ of $0.16 \mathrm{eV}$. $^{86}$

Many dmit-type complexes containing selenium instead of the sulphur atoms were prepared, ${ }^{7,87,88}$ but only few were studied as neutral complexes. The [Au(dsis) $\left.{ }_{2}\right]$ (dsis: 1,3-diselenole-2-selone4,5-diselenolate, Chart 3) revealed a semiconducting behaviour in a compressed pellet, with a $\sigma_{\mathrm{RT}}$ of $6.3 \times 10^{-4} \mathrm{~S} \mathrm{~cm}^{-1}$ and an $E_{\mathrm{a}}$ of $0.16 \mathrm{eV}^{86}$

A close relative of $\left[\mathrm{Ni}(\mathrm{dmit})_{2}\right]$ is $\left[\mathrm{Ni}(\mathrm{mdt})_{2}\right]$ (mdt: 1,3 -dithiole4,5-dithiolate, Chart 3), ${ }^{89}$ however neither the crystal structure nor the solid-state properties were reported.

The first neutral $\left[\mathrm{M}(\mathrm{dddt})_{2}\right]$ (dddt: 5,6-dihydro-1,4-dithiin2,3-dithiolate, Chart 3) complex studied was with $\mathbf{M}=\mathrm{Au}$. The crystal structure shows the neutral molecules organized in dimers connected by several intermolecular $\mathrm{S} \cdots \mathrm{S}$ contacts $(\sim 3.60 \AA)$ in the three dimensions. In single crystals the $\sigma_{\mathrm{RT}}$ is of $10^{-4} \mathrm{~S} \mathrm{~cm}^{-1} \cdot{ }^{90}\left[\mathrm{Ni}(\mathrm{dddt})_{2}\right]$ and $\left[\mathrm{Pt}(\mathrm{dddt})_{2}\right]$ were found to be isostructural with a crystal structure composed of regular stacks of the neutral molecules. ${ }^{91-93}\left[\mathrm{Ni}(\mathrm{dddt})_{2}\right]$ displays a semiconducting behaviour under pressure, with a $\sigma_{\mathrm{RT}}$ ranging from $1.6 \times$ $10^{-3} \mathrm{~S} \mathrm{~cm}^{-1}\left(E_{\mathrm{a}}=0.14 \mathrm{eV}\right)$ at $5.9 \mathrm{GPa}$ to $1.1 \mathrm{~S} \mathrm{~cm}^{-1}\left(E_{\mathrm{a}}=\right.$ $0.046 \mathrm{eV}$ ) at $21.6 \mathrm{GPa}$, and a resistivity behaviour independent of pressure above $9.5 \mathrm{GPa} .{ }^{94}$ In the case of the $\left[\mathrm{Pd}(\mathrm{dddt})_{2}\right]$ complex it also crystallizes in uniform stacks with two crystallographically independent molecular layers. A decrease of resistivity as the pressure increases can be seen, and at $12.6 \mathrm{GPa}$ a temperatureindependent resistivity and the appearance of a Dirac electron system can be also observed. The Dirac cones result from the crossing of the HOMO and LUMO bands that are associated with different molecular layers. ${ }^{30,31}$

Alkyl substituents were also incorporated in the molecular structure of $\left[\mathrm{M}(\mathrm{dddt})_{2}\right]$, such as methyl, phenyl and pyridyl. Regarding [Ni(phdt) ${ }_{2}$ ] (phdt: 5,6-dihydro-5-phenyl-1,4-dithiin-2,3dithiolate, Chart 3) crystal structure it shows uniform stacks with two different orientations, but with large separation between the stacks due to the phenyl substituents. This can explain the semiconducting behaviour observed in this complex, that revealed a $\sigma_{\mathrm{RT}}$ in compressed pellet of $1.5 \times 10^{-6} \mathrm{~S} \mathrm{~cm}^{-1} \cdot{ }^{95}$ The crystal structure of $\left[\mathrm{Ni}(\text { medt })_{2}\right]$ (medt: 5,6-dihydro-6-methyl1,4-dithiin-2,3-dithiolate, Chart 3), resembles the structure of [Ni(phdt $)_{2}$ ] but with closer stacks, however the conductivity was not reported. ${ }^{96}$ As for the $\left[\mathrm{Au}(4-\mathrm{pdddt})_{2}\right]$ (4-pdddt: 5-(pyridin-4-yl)5-hydro-6-dihydro-1,4-dithiin-2,3-dithiolate, Chart 3) complex, it revealed in a microcrystalline compressed pellet a $\sigma_{\mathrm{RT}}$ of $10^{-8} \mathrm{~S} \mathrm{~cm}^{-1} .97$

Since the discover of SCMM, one of the main goals is the design of multifunctional materials. Envisioning that, chiral [M(dm-dddt $\left.)_{2}\right]$ (dm-dddt: 5,6-dimethyl-5,6-dihydro-1,4-dithiin2,3-dithiolate, $\mathrm{M}$ : Au and Ni, Chart 3) and [Ni(me-dddt) 2 (me-dddt: 5-methyl-5,6-dihydro-1,4-dithiin-2,3-dithiolate, Chart 3) complexes were prepared. The $\left[\mathrm{Au}(\mathrm{dm}-\mathrm{dddt})_{2}\right]$ and $\left[\mathrm{Ni}(R, R-\mathrm{dm}-\mathrm{dddt})_{2}\right]$ are isostructural. Even so the bond lengths analysis suggests that the unpaired electron, in the case of the gold complex, is localized in one of the ligands, while in the case of the Ni complex the

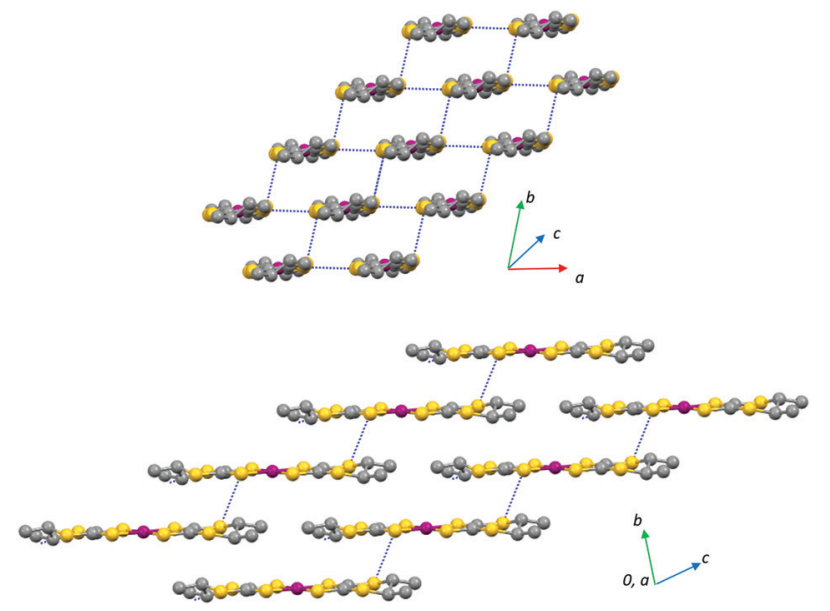

Fig. 16 Detail emphasizing the step-chains like in the crystal structure of $\left[\mathrm{Au}(R, R-\mathrm{dm}-\mathrm{dddt})_{2}\right]$, with blue lines representing $\mathrm{S} \cdots \mathrm{S}$ short contacts.

electron density is uniformly distributed. ${ }^{16,90}$ In the crystal structure, the molecules are organized as step-chains like structures (Fig. 16) with several S. . S contacts (3.67-3.80 A along $c$ and 3.65$3.99 \AA$ along $a$ ) and also $\mathrm{C}-\mathrm{H} \cdots \mathrm{S}$ hydrogen bonding interactions (2.86-2.98 $\mathrm{A})$ connecting each layer. ${ }^{16}$ The single crystal resistivity measurements of $\left[\mathrm{Au}(R, R \text {-dm-dddt })_{2}\right]$ and $\left[\mathrm{Au}(S, S \text {-dm-dddt })_{2}\right]$ revealed semiconducting behaviours and a $\sigma_{\mathrm{RT}}$ of $0.02-$ $0.04 \mathrm{~S} \mathrm{~cm}^{-1}$ with large activation energies. When hydrostatic pressure is applied, the $\sigma_{\mathrm{RT}}$ of the gold complexes increased exponentially and the $E_{\mathrm{a}}$ decreased. The $2 \mathrm{D}$ character of the structure of $\left[\mathrm{Au}(R, R \text {-dm-dddt })_{2}\right]$ does not lead to higher conductivities because the interactions involved are small, which causes the dispersion on the band structure. Moreover, the calculated extended Hückel band structure and first principles DFT calculations show, respectively, a well separated and narrow SOMO and SOMO-1 bands and an unstable hypothetical metallic state due to the localization of the electrons. Structural and electronic effects are responsible for the localization of the SOMO in only one side of the molecule, which leads to the weak interactions and low conductivity. ${ }^{16}$

The neutral complexes $\left[\mathrm{Ni}(\mathrm{me}-\mathrm{dddt})_{2}\right]$ and $\left[\mathrm{Ni}(\mathrm{dm}-\mathrm{dddt})_{2}\right]$ revealed a semiconducting behaviour with a direct band gap, with maximum $\sigma_{\mathrm{RT}}$ obtained at high pressures (10-11 GPa) ranging between 0.05 and $1.7 \mathrm{~S} \mathrm{~cm}^{-1}$ for the monomethyl and between 1.4 and $3.3 \mathrm{~S} \mathrm{~cm}^{-1}$ for the dimethyl substituted. This difference of conductivities in both families is due to stronger intermolecular interactions in [Ni(dm-dddt $\left.)_{2}\right]$, leading to lower activation energies. $^{98}$

Other neutral bis(1,2-dithiolene) based on dddt ligand were prepared however their conductive properties were not studied. ${ }^{99-102}$ In an attempt to improve the transverse interaction, the coordination sulphur atoms in the structure of [Ni(dddt $\left.)_{2}\right]$ were replaced by selenium atoms, giving the $\left[\mathrm{Ni}(\mathrm{ddds})_{2}\right]$ (ddds: 5,6-dihydro-1,4-dithiin-2,3-diselenolate, Chart 3) complex, which is strongly dimerised. ${ }^{103}$ The neutral complex revealed a nonmagnetic state, which could be explained by the dimerisation that leads to a strong antiferromagnetic spin coupling within the dimer. ${ }^{103}$ 
Different ligands with larger fused rings into the 1,2-dithiolene ligand were also prepared. In the neutral $\left[\mathrm{Ni}(\mathrm{pdt})_{2}\right]$ complex (pdt or pddt: 1,3-propanediyldithioethylene-1,2-dithiolate, Chart 3) only the crystal structure was published. ${ }^{104,105}\left[\mathrm{M}\left(\mathrm{F}_{2} \mathrm{pdt}\right)_{2}\right]\left(\mathrm{F}_{2} \mathrm{pdt}\right.$ : 2,2-difluoropropylenedithioethylene-1,2-dithiolate, $\mathrm{M}$ : $\mathrm{Au}$ and $\mathrm{Ni}$, Chart 3) complexes revealed a semiconducting behaviour with a $\sigma_{\mathrm{RT}}$ of $0.05 \mathrm{~S} \mathrm{~cm}^{-1}$ and an $E_{\mathrm{a}}$ of $0.20 \mathrm{eV}$, in the case of the gold complex, while the nickel complex displays an insulating behaviour. ${ }^{106}$ The gold and nickel analogues are not isostructural even if their crystal structures are dominated by an arrangement in parallel columns, separated by the peripheral fluorine segregation. ${ }^{106}$

The neutral gold complex, analogue of $\left[\mathrm{M}(\mathrm{pdt})_{2}\right]$ but substituted with a chiral 4,5-dimethyl-1,3-dioxolane moiety (Chart 3) shows a crystal structure organized in dimers with short intermolecular S. .S contacts (3.57-3.72 $\AA$ ), enfolded by their bulky substituents, which contributes for the isolation of each dimer, suggesting an insulating behaviour. ${ }^{107}$

The $\left[\mathrm{M}\left(\mathrm{dtdt}^{\prime}\right)_{2}\right]$ (dtdt': 5,7-dihydro-1,4,6-trithiin-2,3-dithiolate, $\mathrm{M}$ : Ni and Pt; in the literature this ligand is addressed as dtdt but to avoid confusion with another ligand with the same abbreviation, here it will be designated as dtdt', Chart 3) revealed in compressed pellets a $\sigma_{\mathrm{RT}}$ of $10^{-7}$ and $10^{-3} \mathrm{~S} \mathrm{~cm}^{-1}$, for the nickel and platinum complexes, respectively. ${ }^{108}$

For $\left[\mathrm{Ni}(\mathrm{dpdt})_{2}\right]$ (dpdt: 6-methylene-1,4-dithiepin-2,3-dithiolate, Chart 3) a $\sigma_{\mathrm{RT}}$ of $2.0 \times 10^{-5} \mathrm{~S} \mathrm{~cm}^{-1}$, measured on compressed pellets, a semiconducting behaviour was reported. ${ }^{109}$ In the crystal structure the molecules are arranged in stacks but with only one intrastack S..S short contact (less than $3.70 \AA$ ), which can explain the low conductivity observed.

Other complexes with even larger rings fused in the 1,2dithiolene were synthesized, such as dithiocane rings. A racemic mixture of [Ni(diotte) $\left.)_{2}\right]$ (diotte: 1,3-dioxolane-tetrathiaethylene, Chart 3), revealed a $\sigma_{\mathrm{RT}}$ of $3.6 \times 10^{-8} \mathrm{~S} \mathrm{~cm}^{-1}$. In turn, the enantiomerically pure complexes, $\left[\mathrm{Ni}\{(R, R) \text {-diotte }\}_{2}\right]$ and $[\mathrm{Ni}\{(S, S)$ diotte $\left.\}_{2}\right]$, presented smaller $\sigma_{\mathrm{RT}}$ of $1.7 \times 10^{-8} \mathrm{~S} \mathrm{~cm}^{-1}$ and $1.6 \times$ $10^{-8} \mathrm{~S} \mathrm{~cm}^{-1}$, respectively. No crystal structures were obtained to elucidate about these behaviours. ${ }^{110}$

\section{BTM complexes with benzenic rings}

In the 90's, before the report of the first SCMMs, other neutral BTM complexes exhibiting modest conducting properties started to arise. With fused benzenic rings, $\left[\mathrm{Au}(\mathrm{bdt})_{2}\right]$ (bdt: benzene-1,2dithiolate, Chart 3) presented a crystal structure composed of regular stacks of the neutral molecules, with intra- and interstack short S $\cdots$ S contacts (3.75 ̊̊and $3.66 \AA$, respectively) (Fig. 17). ${ }^{111} \mathrm{~A}$ weak dimerisation in the stack direction was also found at low temperatures. $^{27}\left[\mathrm{Au}(\mathrm{bdt})_{2}\right]$ single crystals revealed a typical semiconductor behaviour, displaying at ambient pressure, a $\sigma_{\mathrm{RT}}$ of $1.1 \times 10^{-3} \mathrm{~S} \mathrm{~cm}^{-1}$ with an $E_{\mathrm{a}}$ of $0.30 \mathrm{eV}$, that slightly increases to $0.67 \times 10^{-3} \mathrm{~S} \mathrm{~cm}^{-1}$ under $0.54 \mathrm{GPa}^{27}$ The $\left[\mathrm{Au}(\mathrm{dmox})_{2}\right]$ (dmox: 4,5-dimethoxy-benzene-1,2-dithiolate, Chart 3) ${ }^{112}$ complex, was also prepared and it displays, in single crystal, a $\sigma_{\mathrm{RT}}$ of $0.11 \mathrm{~S} \mathrm{~cm}^{-1}$ and an $E_{\mathrm{a}}$ of $0.13 \mathrm{eV}$. $^{112}$

Another example of this family of complexes is the $\left[\mathrm{Au}(\mathrm{cbdt})_{2}\right]$ (cbdt: 4-cyanobenzene-1,2-dithiolate, Chart 3), ${ }^{113}$ that in polycrystalline

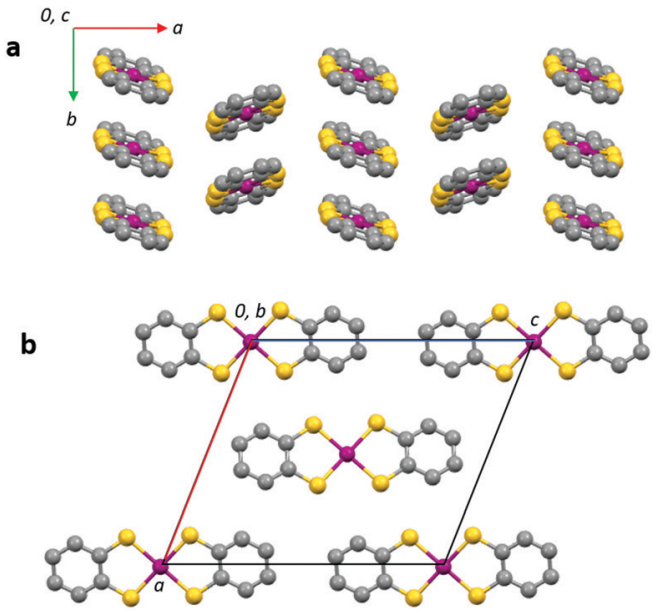

Fig. 17 Crystal structure of $\left[\mathrm{Au}(\mathrm{bdt})_{2}\right]$ : viewed along $c(\mathrm{a})$ and along $b(\mathrm{~b})$.

compressed pellet revealed a semiconductor behaviour with a $\sigma_{\mathrm{RT}}$ of $10^{-4} \mathrm{~S} \mathrm{~cm}^{-1}$.

There are other neutral BTM (M: Au, Ni, Pd and Pt) complexes where the benzene ring was disubstituted with tert-butyl groups, ${ }^{19}$ however they were not studied in the context of SCMM.

\section{Bis(ethylene-1,2-dithiolene) TM complexes with pending units}

Some examples of neutral BTM complexes with ligands based on the 1,2-ethylenedithiolate, bearing pending units can be found in literature. In most cases the transport properties of the neutral complexes were not reported, since these compounds were not prepared with the aim of SCMM. ${ }^{114-132}$ Nevertheless, four cases should be highlighted. The neutral gold complex, with a 4-phenylphenyl pending unit in each ligand (Chart 3), crystallises forming an 1D regular chain with strong short S $\cdots S$ contacts $(<3.70 \AA)$ and in a herringbone packing pattern. ${ }^{133}$ The conductivity at ambient pressure, measured in single crystal ranges between $10^{-4}-10^{-5} \mathrm{~S} \mathrm{~cm}^{-1}$ and a semiconducting behaviour with an $E_{\mathrm{a}}$ of $0.11 \mathrm{eV}$ is observed. However, the band calculations predict a metallic behaviour, which is probably due to a limitation of the extended Hückel theory, since the observed experimental behaviour could be described as a Mott insulator. ${ }^{133}$ There is another example of a neutral gold complex with only a 4-chlorophenyl

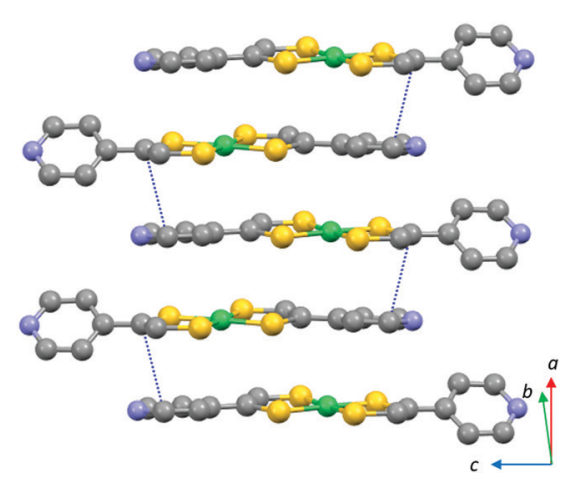

Fig. 18 Details in the crystal structure of [Ni(4-pedt $)_{2}$ ] molecular head-totail stacking, viewed parallel to the central molecular plane. 
Table 1 Summary of the room temperature conductivities $\left(\sigma_{\mathrm{RT}}\right)$ of the neutral BTM complexes discussed. Unless stated otherwise it is assumed measurements in single crystals (*: in compressed pellet; a: sample obtained by iodine oxidation, b: sample obtained by air exposure, c: poor quality crystals and d: better quality crystals, M: metallic, SC: semiconducting, SM: semimetallic, In: insulator, SupC: superconducting, a.p.: ambient pressure)

\begin{tabular}{|c|c|c|c|}
\hline Complex & $\sigma_{\mathrm{RT}}, \mathrm{S} \mathrm{cm} \mathrm{cm}^{-1}\left(E_{\mathrm{a}}, \mathrm{meV}\right)$ & Behaviour & Ref. \\
\hline \multicolumn{4}{|l|}{ TTF-extended ligands } \\
\hline$\left[\mathrm{Hg}(\mathrm{dttfdt})_{2}\right](\mathrm{R}: \mathrm{Et})$ & $10^{-5}$ & $\mathrm{SC}$ & 35 and 36 \\
\hline$\left[\mathrm{Ni}(\mathrm{dttfdt})_{2}\right](\mathrm{R}: \mathrm{Me})$ & $10^{-1}(90)^{*}$ & $\begin{array}{l}300-275 \mathrm{~K}: \mathrm{M} \\
<275 \mathrm{~K}: \mathrm{SC}\end{array}$ & 28 and 35 \\
\hline$\left[\mathrm{Ni}(\mathrm{dttfdt})_{2}\right]$ (R: Et) & $2.7 \times 10^{-2}(100)^{*}$ & SC & 28 and 35 \\
\hline$\left[\mathrm{Ni}(\mathrm{dttfdt})_{2}\right](\mathrm{R}: \mathrm{Bu})$ & $10^{-4}(230)^{*}$ & SC & 28 \\
\hline$\left[\mathrm{Pd}(\mathrm{C} 3-\mathrm{tdt})_{2}\right]$ & $10^{-2}(11-34)$ & SC & 37 \\
\hline$\left[\mathrm{Ni}(\mathrm{tmdt})_{2}\right]$ & 400 & $\mathbf{M}$ & 11 \\
\hline$\left[\mathrm{Au}(\mathrm{tmdt})_{2}\right]$ & $\begin{array}{l}15(20)^{*} \\
50(16)^{*}\end{array}$ & M & 14,39 and 40 \\
\hline$\left[\mathrm{Pt}(\mathrm{tmdt})_{2}\right]$ & $350^{*}$ & M & 41 \\
\hline$\left[\mathrm{Cu}(\mathrm{tmdt})_{2}\right]$ & $7 ; 54(150-250 \mathrm{~K})^{*}$ & $\mathrm{SC}$ & 42 \\
\hline$\left[\mathrm{Co}(\mathrm{tmdt})_{2}\right]$ & $1.5(24)$ & SC & 20 \\
\hline$\left[\mathrm{Pd}(\mathrm{tmdt})_{2}\right]$ & $100(3-9)$ & $\begin{array}{l}\text { SM near RT } \\
\text { SC at lower T }\end{array}$ & 43 \\
\hline$\left[\mathrm{Zn}(\mathrm{tmdt})_{2}\right]$ & $2 \times 10^{-2}(150)^{*}$ & SC & 44 \\
\hline$\left[\mathrm{Ni}(\mathrm{dmdt})_{2}\right]$ & $300-400$ & M & 29 \\
\hline$\left[\mathrm{Au}(\mathrm{dmdt})_{2}\right]$ & $12(9)^{*}$ & $\begin{array}{l}>50 \mathrm{~K}: \mathrm{M} \\
<50 \mathrm{~K}: \mathrm{SC}\end{array}$ & 40 \\
\hline$\left[\mathrm{Cu}(\mathrm{dmdt})_{2}\right]$ & a.p.: $3(40)^{\mathrm{c}}$ and $110^{\mathrm{d}}$ & $\begin{array}{l}\mathrm{SC}^{\mathrm{c}}, \mathrm{M} \text { near } \mathrm{RT}^{\mathrm{d}} \\
\text { High pressure: } \mathrm{M}^{\mathrm{d}}\end{array}$ & 45 and 46 \\
\hline$\left[\mathrm{Pd}(\mathrm{dmdt})_{2}\right]$ & 150 & $\mathrm{SC}$ & 20 \\
\hline$\left[\mathrm{Zn}(\mathrm{dmdt})_{2}\right]$ & $0.5 \times 10^{-2}(160)^{*}$ & SC & 44 \\
\hline$\left[\mathrm{Fe}(\mathrm{dmdt})_{2}\right]$ & $10^{-2}(136)$ & SC & 48 \\
\hline$\left[\mathrm{Co}(\mathrm{dmdt})_{2}\right]$ & $1.2 \times 10^{-2}(85)$ & SC & 49 \\
\hline$\left[\mathrm{Pt}(\mathrm{dmdt})_{2}\right]$ & $150^{*}$ & SM & 32 \\
\hline$\left[\mathrm{Ni}(\mathrm{chdt})_{2}\right]$ & $2(38)^{*}$ & SC & 20 and 50 \\
\hline$\left[\mathrm{Ni}(\text { eodt })_{2}\right]$ & 8 & $>120 \mathrm{~K}: \mathrm{M}$ & 20 and 50 \\
\hline \multirow[t]{2}{*}[\mathrm{Ni}(\mathrm{hfdt})_{2}]{} & $1.6 \times 10^{-3}(140)$ & SC & 51 \\
\hline & & 7.5-8.6 GPa: SupC & \\
\hline$\left[\mathrm{Au}(\mathrm{hfdt})_{2}\right]$ & $3.2 \times 10^{-3 *}(120)$ & SC & 51 \\
\hline$\left[\mathrm{Pd}(\mathrm{hfdt})_{2}\right]$ & $3.7 \times 10^{-3}(160)$ & SC & 53 \\
\hline$\left[\mathrm{Ni}(\mathrm{ptdt})_{2}\right]$ & a.p.: 7 (3) & $\begin{array}{l}\text { a.p.: SC } \\
19 \text { GPa: M }\end{array}$ & 55 and 56 \\
\hline$\left[\mathrm{Au}(\mathrm{ptdt})_{2}\right]$ & $2(18-28)$ & M & 57 \\
\hline$\left[\mathrm{Ni}(\mathrm{dt})_{2}\right]$ & $16(35)$ & SC & 20 \\
\hline$\left[\mathrm{Co}(\mathrm{dt})_{2}\right]$ & $19^{*}$ & $\mathbf{M}$ & 49 and 59 \\
\hline$\left[\mathrm{Pd}(\mathrm{dt})_{2}\right]$ & $0.3(94)^{*}$ & SC & 58 \\
\hline$\left[\mathrm{Zn}(\mathrm{dt})_{2}\right]$ & $0.5 \times 10^{-3}(110)^{*}$ & SC & 44 \\
\hline$\left[\mathrm{Ni}(\mathrm{btdt})_{2}\right]$ & 10 & narrow-gap SC & 60 \\
\hline$\left[\mathrm{Au}(\mathrm{dtdt})_{2}\right]$ & $8(<20)^{* a}$ & SC & 61 \\
\hline \multirow[t]{2}{*}[\mathrm{Ni}(\mathrm{dtdt})_{2}]{} & $2.5(<20)^{* a}$ & $\mathrm{SC}^{\mathrm{a}}$ & 61 \\
\hline & $200(<20)^{* b}$ & $\mathrm{M}^{\mathrm{b}}$ & \\
\hline$\left[\mathrm{Au}(\alpha-\mathrm{tdt})_{2}\right]$ & $5(<20)^{* a}$ & $\mathrm{SC}$ & 61 \\
\hline \multirow{2}{*}[\mathrm{Ni}(\alpha-\mathrm{tdt})_{2}]{} & $2(<20)^{* a}$ & $\mathrm{SC}^{\mathrm{a}}$ & 61 \\
\hline & $24(<20)^{* \mathrm{~b}}$ & $\mathrm{M}^{\mathrm{b}}$ & \\
\hline \multirow[t]{2}{*}[\mathrm{Ni}(\alpha-\mathrm{mtdt})_{2}]{} & $4.2(20)^{\mathrm{a}}$ & SC & 26 \\
\hline & $3.2(33)^{\mathrm{b}}$ & & \\
\hline$\left[\mathrm{Au}(\alpha-\mathrm{mtdt})_{2}\right]$ & $0.32(54)^{* a}$ & SC & 26 \\
\hline$\left[\mathrm{Ni}(\mathrm{tmstfdt})_{2}\right]$ & $100^{*}$ & $\mathbf{M}$ & 64 \\
\hline$\left[\mathrm{Au}(\mathrm{tmstfdt})_{2}\right]$ & $11(28)^{*}$ & $\mathrm{SC}$ & 64 \\
\hline$\left[\mathrm{Au}(\text { pesdt })_{2}\right]$ & $<10^{-8 *}$ & In & 65 \\
\hline \multicolumn{4}{|c|}{ Thiophene/selenophene ligands } \\
\hline$\left[\mathrm{Au}(\alpha-\mathrm{tpdt})_{2}\right]$ & $6^{*}$ & M & 12 \\
\hline$\left[\mathrm{Au}(\mathrm{dtpdt})_{2}\right]$ & $1 \times 10^{-9 *}$ & SC & 12 \\
\hline$\left[\mathrm{Au}(\mathrm{tpdt})_{2}\right]$ & $3.3 \times 10^{-6 *}$ & $\mathrm{SC}$ & 12 \\
\hline$\left[\mathrm{Ni}(\alpha-\mathrm{mtpdt})_{2}\right]$ & $5.2 \times 10^{-7}(325)^{*}$ & SC & 68 \\
\hline$\left[\mathrm{Au}(\alpha-\mathrm{mtpdt})_{2}\right]$ & $8.7 \times 10^{-5}(287)^{*}$ & SC & 68 \\
\hline$\left[\mathrm{Au}(\alpha-\mathrm{dp} \text {-tpdt })_{2}\right]$ & $10^{-8}-5 \times 10^{-5}$ & In & 69 \\
\hline$\left[\mathrm{Au}(\alpha-\mathrm{tp}-\mathrm{tpdt})_{2}\right]$ & $10^{-8}-5 \times 10^{-5}$ & In & 69 \\
\hline$\left[\mathrm{Ni}(\alpha-\mathrm{dp} \text {-tpdt })_{2}\right]$ & $10^{-8}-5 \times 10^{-5}$ & In & 69 \\
\hline$\left[\mathrm{Ni}(\alpha-\mathrm{tp} \text {-tpdt })_{2}\right]$ & $10^{-8}-5 \times 10^{-5}$ & In & 69 \\
\hline$\left[\mathrm{Au}(\alpha \text {-EtMe-tpdt })_{2}\right]$ & $3 \times 10^{-3}(150)$ & Mott In & 70 \\
\hline$\left[\mathrm{Au}\left(\alpha-\mathrm{Me}_{2}-\mathrm{tpdt}\right)_{2}\right]$ & $0.01(120)^{*}$ & SC & 70 \\
\hline$\left[\mathrm{Au}(\mathrm{dspdt})_{2}\right]$ & $0.1(95)$ & SC & 71 \\
\hline
\end{tabular}


Table 1 (continued)

\begin{tabular}{|c|c|c|c|}
\hline Complex & $\sigma_{\mathrm{RT}}, \mathrm{S} \mathrm{cm}{ }^{-1}\left(E_{\mathrm{a}}, \mathrm{meV}\right)$ & Behaviour & Ref. \\
\hline \multicolumn{4}{|l|}{ Thiazole ligands } \\
\hline \multirow{2}{*}[\mathrm{Au}(\text{Et-thiazdt})_{2}]{} & $0.33(120)$ & SM & 21 \\
\hline & 21 kbar: 1000 & $>13$ kbar: $M$ & \\
\hline$\left[\mathrm{Au}(\text { Et-thiazodt })_{2}\right]$ & $0.33 \times 10^{-2}(180)$ & SC & 73 \\
\hline \multirow{2}{*}[\mathrm{Au}(\text{Et-thiazds})_{2}]{} & $40(50)$ & Mott In & 73 \\
\hline & 12.5 kbar: 2600 & $>6$ kbar: $M$ & \\
\hline$\left[\mathrm{Au}(\text { Et-thiazods })_{2}\right]$ & $0.18(60)$ & SC & 73 \\
\hline mono-[Ni(Et-thiazdt $\left.)_{2}\right]$ & $1.4 \times 10^{-2}(200)$ & SC & 74 \\
\hline \multirow{2}{*}{ tricl-[Ni(Et-thiazdt $\left.)_{2}\right]$} & $4.3 \times 10^{-4}(210)$ & $\mathrm{SC}$ & 75 \\
\hline & & 6.9 GPa: M & \\
\hline$\left[\mathrm{Au}(\mathrm{EtOH} \text {-thiazdt })_{2}\right]$ & $0.05-0.07(140)$ & SM & 76 \\
\hline$\left[\mathrm{Au}(\mathrm{cPr} \text {-thiazdt })_{2}\right]$ & $0.037(140)$ & SM & 78 \\
\hline$\left[\mathrm{Au}(n \text { Pr-thiazdt })_{2}\right]$ & $0.36(70)$ & SM & 78 \\
\hline$\left[\mathrm{Au}(\mathrm{iPr} \text {-thiazdt })_{2}\right]$ & $5(60)$ & SM & 77 \\
\hline$\left[\mathrm{Au}\left(\mathrm{NMe}_{2} \text {-thiazdt }\right)_{2}\right]$ & $0.045(180)$ & SM & 78 \\
\hline$\left[\mathrm{Au}(\mathrm{Me}-\mathrm{thiazdt})_{2}\right]$ & 750 & M & 79 \\
\hline \multirow{2}{*}[\mathrm{Ni}(\text{Me-thiazdt})_{2}]{} & $5 \times 10^{-2}(110)$ & SC & 75 \\
\hline & 19 GPa: 166 & & \\
\hline$\left[\mathrm{Pt}(\text { Me-thiazdt })_{2}\right]$ & $1.6 \times 10^{-2}(160)$ & SC & 75 \\
\hline$\left[\mathrm{Pt}(\text { Et-thiazdt })_{2}\right]$ & $2.9 \times 10^{-3}(180)$ & SC & 75 \\
\hline$\left[\mathrm{Au}(\mathrm{MeS}-\mathrm{tzdt})_{2}\right]$ & $8 \times 10^{-3}(130)$ & SC & 80 \\
\hline$\left[\mathrm{Au}(\text { EtS-tzdt })_{2}\right]$ & $3.3 \times 10^{-4}(120)$ & SC & 80 \\
\hline$\left[\mathrm{Ni}(\mathrm{MeS}-\mathrm{tzdt})_{2}\right]$ & $5 \times 10^{-7}-5 \times 10^{-6}(300)$ & SC & 81 \\
\hline$\left[\mathrm{Ni}(\mathrm{EtS}-\mathrm{tzdt})_{2}\right]$ & $10^{-5}-10^{-4}(240)$ & SC & 81 \\
\hline$\left[\mathrm{Ni}(\text { EtOHS-tzdt })_{2}\right]$ & $1.6 \times 10^{-5}$ & $\mathrm{SC}$ & 82 \\
\hline$\left[\mathrm{Au}(\text { EtOHS-tzdt })_{2}\right]$ & $6 \times 10^{-2}(130)$ & Mott In & 82 \\
\hline$\left[\mathrm{Au}(\mathrm{I}-\mathrm{tzdt})_{2}\right]$ & $4-5 \times 10^{-3 *}$ & SC & 83 \\
\hline \multicolumn{4}{|l|}{ Other sulphur/selenium heterocycles } \\
\hline \multirow[t]{2}{*}[\mathrm{Ni}(\mathrm{dmit})_{2}]{} & a.p.: $3.5 \times 10^{-3}$ & a.p.: SC & 84 and 85 \\
\hline & 25.5 GPa: 140 & $>15.9 \mathrm{GPa}: \mathrm{M}$ & \\
\hline$\left[\mathrm{Au}(\mathrm{dmit})_{2}\right]$ & $5.0 \times 10^{-4}(160)$ & SC & 86 \\
\hline$\left[\mathrm{Au}(\mathrm{dsis})_{2}\right]$ & $6.3 \times 10^{-4 *}(160)$ & SC & 86 \\
\hline$\left[\mathrm{Au}(\mathrm{dddt})_{2}\right]$ & $10^{-4}$ & & 90 \\
\hline \multirow{3}{*}[\mathrm{Ni}(\mathrm{dddt})_{2}]{} & a.p.: - & a.p.: In & 91,92 and 94 \\
\hline & $5.9 \mathrm{GPa}: 1.6 \times 10^{-3}(140)$ & 5.9 GPa: SC & \\
\hline & 21.6 GPa: $1.1(46)$ & $21.6 \mathrm{GPa}: \mathrm{SC}$ & \\
\hline \multirow[t]{2}{*}[\mathrm{Pd}(\mathrm{dddt})_{2}]{} & a.p.: - & a.p.: In & 93 \\
\hline & & 12.6 GPa: M & \\
\hline$\left[\mathrm{Ni}(\mathrm{phdt})_{2}\right]$ & $1.5 \times 10^{-6 *}$ & SC & \\
\hline$\left[\mathrm{Au}(4-\mathrm{pdddt})_{2}\right]$ & $10^{-8 *}$ & $\sim$ In & 97 \\
\hline$\left[\mathrm{Au}(\mathrm{dm} \text {-dddt })_{2}\right]$ & $0.02-0.04$ & SC & 16 \\
\hline$\left[\mathrm{Ni}(\mathrm{dm} \text {-dddt })_{2}\right]$ & 10-11 GPa: $1.4-3.3$ & SC & 98 \\
\hline$\left[\mathrm{Ni}(\mathrm{me}-\mathrm{dddt})_{2}\right]$ & 10-11 GPa: $0.05-1.7$ & SC & 98 \\
\hline$\left[\mathrm{Au}\left(\mathrm{F}_{2} \mathrm{pdt}\right)_{2}\right]$ & $0.05(200)$ & SC & 106 \\
\hline$\left[\mathrm{Ni}\left(\mathrm{F}_{2} \mathrm{pdt}\right)_{2}\right]$ & - & In & \\
\hline$\left[\mathrm{Au}(\mathrm{chiral} \text { 4,5-dimethyl-1,3-dioxolane substituted pdt })_{2}\right]$ & - & In & 107 \\
\hline$\left[\mathrm{Ni}\left(\mathrm{dtdt}^{\prime}\right)_{2}\right]$ & $10^{-7 *}$ & SC & 108 \\
\hline$\left[\operatorname{Pt}\left(\mathrm{dtdt}^{\prime}\right)_{2}\right]$ & $10^{-3 *}$ & SC & 108 \\
\hline$\left[\mathrm{Ni}(\mathrm{dpdt})_{2}\right]$ & $2.0 \times 10^{-5 *}$ & SC & 109 \\
\hline$\left[\mathrm{Ni}(\text { diotte })_{2}\right]$ & $3.6 \times 10^{-8 *}$ & SC & 110 \\
\hline$\left[\mathrm{Ni}\{(R, R) \text {-diotte }\}_{2}\right]$ & $1.7 \times 10^{-8 *}$ & SC & 110 \\
\hline$\left[\mathrm{Ni}\{(S, S) \text {-diotte }\}_{2}\right]$ & $1.6 \times 10^{-8 *}$ & SC & 110 \\
\hline \multicolumn{4}{|l|}{ Benzenic ligands } \\
\hline \multirow{2}{*}[\mathrm{Au}(\mathrm{bdt})_{2}]{} & a.p.: $1.1 \times 10^{-3}(300)$ & SC & 27 \\
\hline & 0.55 GPa: $0.67 \times 10^{-3}$ & & \\
\hline$\left[\mathrm{Au}(\mathrm{cbdt})_{2}\right]$ & $10^{-4 *}$ & SC & 113 \\
\hline$\left[\mathrm{Au}(\mathrm{dmox})_{2}\right]$ & $0.11(130)$ & SC & 112 \\
\hline \multicolumn{4}{|l|}{ Pending ligands } \\
\hline \multirow[t]{2}{*}{$\mathrm{Au}\left(1-((1,1-\mathrm{biphenyl})-4 \text {-yl-)-ethylene-1,2-dithiolene })_{2}\right]$} & $10^{-4}-10^{-5}$ & Mott In & 133 \\
\hline & máx: $1.5 \times 10^{-4}(110)$ & & \\
\hline [Au(1-((1-chlorophenyl)-4-yl-)-ethylene-1,2-dithiolene) $\left.)_{2}\right]$ & $3.06 \times 10^{-7}(250)$ & SC & 134 \\
\hline$\left[\mathrm{Ni}(4-\text { pedt })_{2}\right]$ & $2 \times 10^{-6}$ & SC & 135 \\
\hline$\left[\mathrm{Ni}(\mathrm{dpedt})_{2}\right]$ & $9.47 \times 10^{-6 *}$ & SC & 139 \\
\hline
\end{tabular}


pending in each ligand (Chart 3), that displays a thermally activated behaviour with a $\sigma_{\mathrm{RT}}$ of $3.06 \times 10^{-7} \mathrm{~S} \mathrm{~cm}^{-1}$ and an $E_{\mathrm{a}}$ of $0.25 \mathrm{eV}$. The semiconducting behaviour remains even under $2 \mathrm{GPa}^{134}$

In the $\left[\mathrm{Ni}(4 \text {-pedt })_{2}\right]$ (4-pedt: 1-(pyridine-4-yl)-ethylene-1,2dithiolate, Chart 3), ${ }^{135}$ the crystal structure is composed of stacks organised in a head-to-tail pattern. The pyridine groups are in a trans configuration and with different degrees of rotation out of the plane of the bis(1,2-dithiolene) core (Fig. 18), around $43.1^{\circ}$ due to intermolecular hydrogen bonds and $\pi \cdots \pi$ interactions and $\sim 6.44^{\circ}$ due to sandwiching between the neighbouring complexes. The overlap pattern shows a large longitudinal slid which keeps the metal centers far apart from each other, but close to the bond to the pending group. In single crystals it revealed a poor semiconducting behaviour with a $\sigma_{\mathrm{RT}}$ of $2 \times 10^{-6} \mathrm{~S} \mathrm{~cm}^{-1} .135$

In the case of $\left[\mathrm{Ni}(\mathrm{dpedt})_{2}\right]^{136}$ (dpedt: 1,2-diphenyl-1,2-ethylenedithiolate, Chart 3), as a compressed pellet, displays a semiconductor behaviour with a $\sigma_{\mathrm{RT}}$ of $9.47 \times 10^{-6} \mathrm{~S} \mathrm{~cm}^{-1} \cdot{ }^{137-140}$

A series of BTM complexes with ligands based on the 1,2-ethylenedithiolate, bearing $\mathrm{CF}_{3}$ groups, $\left[\mathrm{M}(\mathrm{tds})_{2}\right]$ (tds: [bis(trifluoromethyl)-ethylene]diselenolate, $\mathrm{M}$ : $\mathrm{Ni}$ and $\mathrm{Pt}$ ) was also prepared, however their properties were not reported. ${ }^{141-143}$

The synthesis of a few other neutral BTM complexes with different ligands can be found in literature, however their transport properties were not reported, since these compounds were prepared in a different context than SCMM. ${ }^{72,144-150}$

Table 1 summarizes the transport properties $\left(\sigma_{\mathrm{RT}}, E_{\mathrm{a}}\right.$ and general behaviour) of the neutral BTM complexes from the six categories discussed in this review.

\section{Conclusions}

Several BTM complexes have been reported along the years but not all were studied within the scope of SCMC and only few were found to display metallic behaviour. After 2001, their study has been intensified when metallic properties were discovered in the $\left[\mathrm{Ni}(\mathrm{tmdt})_{2}\right]$ and $\left[\mathrm{Au}(\alpha-\mathrm{tpdt})_{2}\right]$, two examples of neutral BTM complexes. Currently, these complexes are still, to the best of our knowledge, the best candidates to obtain a SCMM. SCMM can be divided in two main families, one with extended ligands comprised of a fused substituted TTF moiety and another with smaller ligands, such as thiazole or thiophene rings.

Twenty years of research have highlighted some common features. In the family with extended ligands, the studies suggest that the interactions perceived by the TTF moiety are the main responsible for the solid-state properties since the electronic density is essentially on the TTF core and not on its substituents, nor in the TM atom. But the TTF substituents still play an important role, because although not substantially contributing for the molecular overlapping, some bulkier substituent groups can prevent it. Since the metals seem to retain the elemental magnetic properties, multifunctional materials with metallic and interesting magnetic properties are envisage. In the family of neutral BTM complexes with smaller ligands, the solid-state properties are much more sensitive to the ligand and their non-innocent nature. The best ligands seemed to be the ones comprising multiple heteroatoms, such as sulphur or selenium. In this case, they are even more sensitive to the introduction of substituents because even the smaller changes in the molecular geometry can affect drastically the transport properties in the solid-state. Also, the nature of the central TM seems to be determinant in the molecular overlapping patterns. The gold complexes are often much more conductors due to their open shell character.

Obtaining SCMM from neutral BTM complexes remains a challenge since some of these molecular building blocks are not accessible or even stable in the neutral state, and those who are most often face solubility issues. The synthetic routes are rigorous and time-consuming. Nevertheless, if successful, their unconventional properties are a most sought-after reward.

\section{Conflicts of interest}

There are no conflicts to declare.

\section{Acknowledgements}

This work was partially supported by FCT (Portugal) through Contracts UIDB/04349/2020 and PTDC/QUI-QIN/29834/2017, and $\mathrm{PhD}$ grant $\mathrm{PD} / \mathrm{BD} / 135314 / 2017$ of M. F. G. Velho.

\section{Notes and references}

1 J. Ferraris, D. O. Cowan, V. Walatka and J. H. Perlstein, J. Am. Chem. Soc., 1973, 95, 948-949.

2 D. Jérome, A. Mazaud, M. Ribault and K. Bechgaard, J. Phys. Lett., 1980, 41, 95-98.

3 D. Jérome, Chem. Rev., 2004, 104, 5565-5591.

4 R. P. Shibaeva and E. B. Yagubskii, Chem. Rev., 2004, 104, 5347-5378.

5 C. Faulmann and P. Cassoux, in Dithiolene Chemistry: Synthesis, Properties, and Applications, ed. E. I. Stiefel, John Wiley \& Sons, Inc., Hoboken, New Jersey, 2004, vol. 52, pp. 399-489.

6 N. Robertson and L. Cronin, Coord. Chem. Rev., 2002, 227, 93-127.

7 R. Kato, Chem. Rev., 2004, 104, 5319-5346.

8 L. Brossard, M. Ribault, L. Valade and P. Cassoux, Physica $B+C$, 1986, 143, 378-380.

9 E. B. Yagubskii, A. I. Kotov, A. G. Khomenko, L. I. Buravov and A. I. Schegolev, Synth. Met., 1992, 46, 255-259.

10 R. C. Haddon, Nature, 1975, 256, 394-396.

11 H. Tanaka, Y. Okano, H. Kobayashi, W. Suzuki and A. Kobayashi, Science, 2001, 291, 285-287.

12 D. Belo, H. Alves, E. B. Lopes, M. T. Duarte, V. Gama, R. T. Henriques, M. Almeida, A. Pérez-Benítez, C. Rovira and J. Veciana, Chem. - Eur. J., 2001, 7, 511-519.

13 Y. Kobayashi, T. Terauchi, S. Sumi and Y. Matsushita, Nat. Mater., 2017, 16, 109-114.

14 A. Kobayashi, Y. Okano and H. Kobayashi, J. Phys. Soc. Jpn., 2006, 75, 051002. 
15 C. L. Beswick, J. M. Schulman and E. I. Stiefel, in Dithiolene Chemistry: Synthesis, Properties, and Applications, ed. E. I. Stiefel, John Wiley \& Sons, Inc., Hoboken, New Jersey, 1st edn, 2004, pp. 55-110.

16 D. G. Branzea, F. Pop, P. Auban-Senzier, R. Clérac, P. Alemany, E. Canadell and N. Avarvari, J. Am. Chem. Soc., 2016, 138, 6838-6851.

17 V. Bachler, G. Olbrich, F. Neese and K. Wieghardt, Inorg. Chem., 2002, 41, 4179-4193.

18 T. Waters, H. K. Woo, X. Bin Wang and L. S. Wang, J. Am. Chem. Soc., 2006, 128, 4282-4291.

19 K. Ray, T. Weyhermüller, F. Neese and K. Wieghardt, Inorg. Chem., 2005, 44, 5345-5360.

20 A. Kobayashi, E. Fujiwara and H. Kobayashi, Chem. Rev., 2004, 104, 5243-5264.

21 N. Tenn, N. Bellec, O. Jeannin, L. Piekara-Sady, P. AubanSenzier, J. Íñiguez, E. Canadell and D. Lorcy, J. Am. Chem. Soc., 2009, 131, 16961-16967.

22 G. Schukat and E. Fanghänel, Sulfur Rep., 1996, 18, 1-278.

23 D. Belo and M. Almeida, Coord. Chem. Rev., 2010, 254, 1479-1492.

24 N. Svenstrup and J. Becher, Synthesis, 1995, 215-235.

25 N. Svenstrup, K. M. Rasmussen, T. K. Hansen and J. Becher, Synthesis, 1994, 809-812.

26 R. A. L. Silva, B. J. C. Vieira, M. M. Andrade, I. C. Santos, S. Rabaça, E. B. Lopes, J. T. Coutinho, L. C. J. Pereira, M. Almeida and D. Belo, Molecules, 2018, 23, 1-15.

27 N. C. Schiødt, T. Bjørnholm, K. Bechgaard, J. Neumeier and C. Allgeier, Phys. Rev. B: Condens. Matter Mater. Phys., 1996, 53, 1773-1778.

28 N. Le Narvor, N. Robertson, T. Weyland, J. D. Kilburn, A. E. Underhill, M. Webster, N. Svenstrupd and J. Becherd, Chem. Commun., 1996, 1363-1364.

29 A. Kobayashi, H. Tanaka and H. Kobayashi, J. Mater. Chem., 2001, 11, 2078-2088.

30 R. Kato, H. Cui, T. Tsumuraya, T. Miyazaki and Y. Suzumura, J. Am. Chem. Soc., 2017, 139, 1770-1773.

31 R. Kato, H. Cui, T. Minamidate, H. H. M. Yeung and Y. Suzumura, J. Phys. Soc. Jpn., 2020, 89, 124706.

32 B. Zhou, S. Ishibashi, T. Ishii, T. Sekine, R. Takehara, K. Miyagawa, K. Kanoda, E. Nishibori and A. Kobayashi, Chem. Commun., 2019, 55, 3327-3330.

33 R. Kato and Y. Suzumura, J. Phys. Soc. Jpn., 2020, 89, 044713. 34 T. Kawamura, D. Ohki, B. Zhou, A. Kobayashi and A. Kobayashi, J. Phys. Soc. Jpn., 2020, 89, 074704.

35 A. E. Underhill, N. Robertson, J. Ziegenbalg, N. Le Narvor, J. D. Kilburn and K. Awaga, Mol. Cryst. Liq. Cryst., 1996, 284, 39-48.

36 N. Le Narvor, N. Robertson, E. Wallace, J. D. Kilburn, A. E. Underhill, P. N. Bartlett and M. Webster, J. Chem. Soc., Dalton Trans., 1996, 823-828.

37 W. Suzuki, E. Fujiwara, A. Kobayashi, A. Hasegawa, T. Miyamoto and H. Kobayashi, Chem. Lett., 2002, 936-937.

38 H. Tanaka, M. Tokumoto, S. Ishibashi, D. Graf, E. S. Choi, J. S. Brooks, S. Yasuzuka, Y. Okano and H. Kobayashi, J. Am. Chem. Soc., 2004, 126, 10518-10519.
39 B. Zhou, M. Shimamura, E. Fujiwara, A. Kobayashi, T. Higashi, E. Nishibori, M. Sakata, H. B. Cui, K. Takahashi and H. Kobayashi, J. Am. Chem. Soc., 2006, 128, 3872-3873.

40 W. Suzuki, E. Fujiwara, A. Kobayashi, Y. Fujishiro, E. Nishibori, M. Takata, M. Sakata, H. Fujiwara and H. Kobayashi, J. Am. Chem. Soc., 2003, 125, 1486-1487.

41 B. Zhou, A. Kobayashi, Y. Okano, T. Nakashima, S. Aoyagi, E. Nishibori, M. Sakata, M. Tokumoto and H. Kobayashi, Adv. Mater., 2009, 21, 3596-3600.

42 B. Zhou, H. Yajima, A. Kobayashi, Y. Okano, H. Tanaka, T. Kumashiro, E. Nishibori, H. Sawa and H. Kobayashi, Inorg. Chem., 2010, 49, 6740-6747.

43 S. Ogura, Y. Idobata, B. Zhou, A. Kobayashi, R. Takagi, K. Miyagawa, K. Kanoda, H. Kasai, E. Nishibori, C. Satoko and B. Delley, Inorg. Chem., 2016, 55, 7709-7716.

44 K. Yamamoto, E. Fujiwara, A. Kobayashi, Y. Fujishiro, E. Nishibori, M. Sakata, M. Takata, H. Tanaka, Y. Okano and H. Kobayashi, Chem. Lett., 2005, 34, 1090-1091.

45 H. Tanaka, H. Kobayashi and A. Kobayashi, J. Am. Chem. Soc., 2002, 124, 10002-10003.

46 B. Zhou, Y. Idobata, A. Kobayashi, H. Cui, R. Kato, R. Takagi, K. Miyagawa, K. Kanoda and H. Kobayashi, J. Am. Chem. Soc., 2012, 134, 12724-12731.

47 H. Tanaka, H. Kobayashi and A. Kobayashi, Mol. Cryst. Liq. Cryst., 2002, 380, 197-202.

48 B. Zhou, S. Ogura, N. Kato, Y. Idobata, A. Kobayashi and H. Kobayashi, Chem. Lett., 2013, 42, 977-979.

49 E. Fujiwara, K. Hosoya, A. Kobayashi, H. Tanaka, M. Tokumoto, Y. Okano, H. Fujiwara, H. Kobayashi, Y. Fujishiro, E. Nishibori, M. Takata and M. Sakata, Inorg. Chem., 2008, 47, 863-874.

50 E. Fujiwara, A. Kobayashi, H. Fujiwara and H. Kobayashi, Inorg. Chem., 2004, 43, 1122-1129.

51 M. Sasa, E. Fujiwara, A. Kobayashi, S. Ishibashi, K. Terakura, Y. Okano, H. Fujiwara and H. Kobayashi, J. Mater. Chem., 2005, 15, 155-163.

52 N. M. Rivera, E. M. Engler and R. R. Schumaker, J. Chem. Soc., Chem. Commun., 1979, 184-185.

53 B. Zhou, S. Ogura, Q. Z. Liu, H. Kasai, E. Nishibori and A. Kobayashi, Chem. Lett., 2016, 45, 303-305.

54 H. Cui, H. Kobayashi, S. Ishibashi, M. Sasa, F. Iwase, R. Kato and A. Kobayashi, J. Am. Chem. Soc., 2014, 136, 7619-7622.

55 A. Kobayashi, H. Tanaka, M. Kumasaki, H. Torii, B. Narymbetov and T. Adachi, J. Am. Chem. Soc., 1999, 121, 10763-10771.

56 H. Cui, J. S. Brooks, A. Kobayashi and H. Kobayashi, J. Am. Chem. Soc., 2009, 131, 6358-6359.

57 B. Zhou, H. Yajima, Y. Idobata, A. Kobayashi, T. Kobayashi, E. Nishibori, H. Sawa and H. Kobayashi, Chem. Lett., 2012, 41, 154-156.

58 W. Suzuki, E. Fujiwara, A. Kobayashi, Y. Fujishiro, E. Nishibori, M. Takata, M. Sakata, Y. Okano and H. Kobayashi, Chem. Lett., 2003, 32, 1106-1107.

59 A. Kobayashi, E. Fujiwara, W. Suzuki, M. Sasa, Y. Fujishiro, E. Nishibori, M. Takata, M. Sakata, Y. Okano, H. Fujiwara and H. Kobayashi, J. Phys. IV, 2004, 114, 419-424.

60 H. R. Wen, C. H. Li, Y. Song, J. L. Zuo, B. Zhang and X. Z. You, Inorg. Chem., 2007, 46, 6837-6839. 
61 J. P. M. Nunes, M. J. Figueira, D. Belo, I. C. Santos, B. Ribeiro, E. B. Lopes, R. T. Henriques, J. Vidal-Gancedo, J. Veciana, C. Rovira and M. Almeida, Chem. - Eur. J., 2007, 13, 9841-9849.

62 H. Alves, A. I. S. Neves, W. Gouveia, R. A. L. Silva and D. Belo, Chem. Commun., 2015, 51, 13117-13119.

63 A. I. S. Neves, E. B. Lopes, M. Almeida and D. Belo, Phys. Status Solidi C, 2012, 9, 1137-1139.

64 E. Fujiwara, B. Zhou, A. Kobayashi, H. Kobayashi, Y. Fujishiro, E. Nishibori, M. Sakata, S. Ishibashi and K. Terakura, Eur. J. Inorg. Chem., 2009, 1585-1591.

65 S. I. G. Dias, S. Rabaça, I. C. Santos, L. C. J. Pereira, R. T. Henriques and M. Almeida, Inorg. Chem. Commun., 2012, 15, 102-105.

66 S. Rabaça, S. Oliveira, D. Belo, I. C. Santos and M. Almeida, Inorg. Chem. Commun., 2015, 58, 87-90.

67 E. Laukhina, V. Lebedev, V. Laukhin, A. Pérez, E. B. Lopes, A. I. S. Neves, D. Belo, M. Almeida, J. Veciana and C. Rovira, Org. Electron., 2012, 13, 894-898.

68 A. I. S. Neves, I. C. Santos, J. T. Coutinho, L. C. J. Pereira, R. T. Henriques, E. B. Lopes, H. Alves, M. Almeida and D. Belo, Eur. J. Inorg. Chem., 2014, 3989-3999.

69 M. M. Andrade, R. A. L. Silva, I. C. Santos, E. B. Lopes, S. Rabaça, L. C. J. Pereira, J. T. Coutinho, J. P. Telo, C. Rovira, M. Almeida and D. Belo, Inorg. Chem. Front., 2017, 4, 270-280.

70 T. Higashino, O. Jeannin, T. Kawamoto, D. Lorcy, T. Mori and M. Fourmigué, Inorg. Chem., 2015, 54, 9908-9913.

71 M. F. G. Velho, R. A. L. Silva, G. Brotas, E. B. Lopes, I. C. Santos, A. Charas, D. Belo and M. Almeida, Dalton Trans., 2020, 49, 13737-13743.

72 M. C. Aragoni, M. Arca, F. A. Devillanova, F. Isaia, V. Lippolis, A. Mancini, L. Pala, A. M. Z. Slawin and J. D. Woollins, Inorg. Chem., 2005, 44, 9610-9612.

73 G. Yzambart, N. Bellec, G. Nasser, O. Jeannin, T. Roisnel, M. Fourmigué, P. Auban-Senzier, I. Jorge, E. Canadell and D. Lorcy, J. Am. Chem. Soc., 2012, 134, 17138-17148.

74 A. Filatre-Furcate, N. Bellec, O. Jeannin, P. Auban-Senzier, M. Fourmigué, A. Vacher and D. Lorcy, Inorg. Chem., 2014, 53, 8681-8690.

75 H. Hachem, H. Cui, T. Tsumuraya, R. Kato, O. Jeannin, M. Fourmigué and D. Lorcy, J. Mater. Chem. C, 2020, 8, 11581-11592.

76 Y. Le Gal, T. Roisnel, P. Auban-Senzier, T. Guizouarn and D. Lorcy, Inorg. Chem., 2014, 53, 8755-8761.

77 A. Filatre-furcate, N. Bellec, O. Jeannin, P. Auban-senzier, M. Fourmigué, E. Canadell, B. Brie, V. T. Phuoc and D. Lorcy, Inorg. Chem., 2016, 55, 6036-6046.

78 A. Filatre-Furcate, T. Roisnel, M. Fourmigué, O. Jeannin, N. Bellec, P. Auban-Senzier and D. Lorcy, Chem. - Eur. J., 2017, 23, 16004-16013.

79 Y. Le Gal, T. Roisnel, P. Auban-Senzier, N. Bellec, J. Íñiguez, E. Canadell and D. Lorcy, J. Am. Chem. Soc., 2018, 140, 6998-7004.

80 A. Filatre-Furcate, P. Auban-Senzier, M. Fourmigué, T. Roisnel, V. Dorcet and D. Lorcy, Dalton Trans., 2015, 44, 15683-15689.
81 H. Hachem, Z. Xu, N. Bellec, O. Jeannin, P. Auban-senzier, T. Guizouarn, M. Fourmigué and D. Lorcy, Dalton Trans., 2018, 47, 6580-6589.

82 H. Hachem, N. Bellec, M. Fourmigué and D. Lorcy, Dalton Trans., 2020, 49, 6056-6064.

83 H. Hachem, O. Jeannin, M. Fourmigué, F. Barrière and D. Lorcy, CrystEngComm, 2020, 22, 3579-3587.

84 L. Valade, J. Legros, M. Bousseau, P. Cassoux, M. Garbauskas and V. Leonard, J. Chem. Soc., Dalton Trans., 1985, 783-794.

85 H. Cui, T. Tsumuraya, T. Miyazaki, Y. Okano and R. Kato, Eur. J. Inorg. Chem., 2014, 3837-3840.

86 G. Matsubayashi and A. Yokozawa, J. Chem. Soc., Dalton Trans., 1990, 3535-3539.

87 R. Kato, Bull. Chem. Soc. Jpn., 2014, 87, 355-374.

88 R.-M. Olk, E. Hoyer, C. Faulmann and P. Cassoux, Synth. Met., 1993, 55-57, 2453-2458.

89 Y. S. J. Veldhuizen, N. Veldman, A. L. Spek, P. Cassoux, R. Carlier, M. J. J. Mulder, J. G. Haasnoot and J. Reedijk, J. Chem. Soc., Dalton Trans., 1998, 2989-2993.

90 A. J. Schultz, H. H. Wang, H. W. Lynda, T. L. Sifter, J. M. Williams, K. Bechgaard and M. Whangbo, Inorg. Chem., 1987, 26, 3757-3761.

91 H. Kim, A. Kobayashi, Y. Sasaki, R. Kato and H. Kobayashi, Bull. Chem. Soc. Jpn., 1988, 61, 579-581.

92 S. S. Nahapetyan, V. E. Shklover, L. V. Vetoshkina, A. I. Kotov, L. Y. Ukhin, Y. T. Struchkov and E. B. Yagubskii, Mater. Sci., 1988, 14, 5-9.

93 V. V. Gritsenko, O. A. D’yachenko, P. Cassoux, A. I. Kotov, E. E. Laukhina, C. Faulmann and E. B. Yagubskii, Russ. Chem. Bull., 1993, 42, 1149-1151.

94 H. Cui, T. Tsumuraya, H. H.-M. Yeung, C. S. Coates, M. R. Warren and R. Kato, Molecules, 2019, 24, 1843.

95 J.-L. Zuo, T.-M. Yao, F. You, X.-Z. You, H.-K. Fun and B.-C. Yip, J. Mater. Chem., 1996, 6, 1633-1637.

96 J. L. Zuo, F. You, X. Z. You and H. K. Fun, Polyhedron, 1997, 16, 1465-1469.

97 S. Oliveira, M. L. Afonso, S. I. G. Dias, I. C. Santos, R. T. Henriques, S. Rabaça and M. Almeida, Eur. J. Inorg. Chem., 2013, 3133-3136.

98 A. Abhervé, N. Mroweh, T. Cauchy, F. Pop, H. Cui, R. Kato, N. Vanthuyne, P. Alemany, E. Canadell and N. Avarvari, J. Mater. Chem. C, 2021, 9, 4119-4140.

99 G. Soras, N. Psaroudakis, G. A. Mousdis, M. J. Manos, A. J. Tasiopoulos, P. Aloukos, S. Couris, P. Labéguerie, J. Lipinski, A. Avramopoulos and M. G. Papadopoulos, Chem. Phys., 2010, 372, 33-45.

100 G. Soras, N. Psaroudakis, M. J. Manos, A. J. Tasiopoulos, D. G. Liakos and G. A. Mousdis, Polyhedron, 2013, 62, 208-217.

101 G. Soras, N. Psaroudakis, M. J. Manos, A. J. Tasiopoulos, D. G. Liakos and G. A. Mousdis, Polyhedron, 2009, 28, 3340-3348.

102 L. Hu, J. Qin, N. Zhou, Y. F. Meng, Y. Xu, J. L. Zuo and X. Z. You, Dyes Pigm., 2012, 92, 1223-1230.

103 H. Fujiwara, E. Ojima, H. Kobayashi, T. Courcet, I. Malfant and P. Cassoux, Eur. J. Inorg. Chem., 1998, 1631-1639. 
104 R. Kato, H. Kobayashi, A. Kobayashi and Y. Sasaki, Bull. Chem. Soc. Jpn., 1986, 59, 627-630.

105 U. Geiser, S. F. Tytko, T. J. Allen, H. H. Wang, A. M. Kini and J. M. Williams, Acta Crystallogr., Sect. C: Cryst. Struct. Commun., 1991, 47, 1164-1167.

106 O. J. Dautel, M. Fourmigué, E. Canadell and P. AubanSenzier, Adv. Funct. Mater., 2002, 12, 693-698.

107 R. Le Pennec, O. Jeannin, P. Auban-Senzier and M. Fourmigué, New J. Chem., 2016, 40, 7113-7120.

108 C. Faulmann, A. Errami, B. Donnadieu, I. Malfant, J. Legros, P. Cassoux, C. Rovira and E. Canadell, Inorg. Chem., 1996, 35, 3856-3873.

109 A. Charlton, A. E. Underhill, K. M. A. Malik, M. B. Hursthouse, T. Jcrgensen and J. Becher, Synth. Met., 1995, 68, 221-226.

110 H. Kisch, B. Eisen, R. Dinnebier, K. Shankland, W. I. F. David and F. Knoch, Chem. - Eur. J., 2001, 7, 738-748.

111 G. Rindorf, N. Thorup, T. Bjørnholm and K. Bechgaard, Acta Crystallogr. Sect. C Cryst. Struct. Commun., 1990, 46, 1437-1439.

112 N. C. Schiødt, T. Bjørnholm, C. S. Jacobsen and K. Bechgaard, Synth. Met., 1993, 56, 2164-2168.

113 A. C. Cerdeira, M. L. Afonso, I. C. Santos, L. C. J. Pereira, J. T. Coutinho, S. Rabaça, D. Simão, R. T. Henriques and M. Almeida, Polyhedron, 2012, 44, 228-237.

114 R. Perochon, L. Piekara-Sady, W. Jurga, R. Clérac and M. Fourmigué, Dalton Trans., 2009, 3052-3061.

115 S. Kokatam, K. Ray, J. Pap, E. Bill, W. E. Geiger, R. J. LeSuer, P. H. Rieger, T. Weyhermüller, F. Neese and K. Wieghardt, Inorg. Chem., 2007, 46, 1100-1111.

116 C. L. Kean and P. G. Pickup, Chem. Commun., 2001, 815-816.

117 T. Anjos, S. J. Roberts-Bleming, A. Charlton, N. Robertson, A. R. Mount, S. J. Coles, M. B. Hursthouse, M. Kalaji and P. J. Murphy, J. Mater. Chem., 2008, 18, 475-483.

118 A. Sournia-Saquet, B. G. de Bonneval, K. I. Chane-Ching and L. Valade, J. Electroanal. Chem., 2008, 624, 84-90.

119 S. Dalgleish and N. Robertson, Chem. Commun., 2009, 5826-5828.

120 C. A. S. Hill, A. Charlton, A. E. Underhill, K. M. A. Malik, M. B. Hursthouse, A. I. Karaulov, S. N. Oliver and S. V. Kershaw, J. Chem. Soc., Dalton Trans., 1995, 587-594.

121 A. M. Giroud, A. Nazzal and U. T. Mueller-westerhoff, Mol. Cryst. Liq. Cryst., 1980, 56, 225-228.

122 A. Pintus, L. Ambrosio, M. C. Aragoni, M. Binda, S. J. Coles, M. B. Hursthouse, F. Isaia, V. Lippolis, G. Meloni, D. Natali, J. B. Orton, E. Podda, M. Sampietro and M. Arca, Inorg. Chem., 2020, 59, 6410-6421.

123 U. T. Mueller-Westerhoff, A. Nazzal, R. J. Cox and A. M. Giroud, Mol. Cryst. Liq. Cryst., 1980, 56, 249-255.

124 V. Madhu and S. K. Das, Inorg. Chem., 2008, 47, 5055-5070.

125 K. M. Sung and R. H. Holm, J. Am. Chem. Soc., 2002, 124, 4312-4320.

126 K. Arumugam, J. E. Bollinger, M. Fink and J. P. Donahue, Inorg. Chem., 2007, 46, 3283-3288.
127 G. C. Papavassiliou, G. C. Anyfantis, C. P. Raptopoulou, V. Psycharis, N. Ioannidis, V. Petrouleas and P. Paraskevopoulou, Polyhedron, 2009, 28, 3368-3372.

128 Q. Miao, J. Gao, Z. Wang, H. Yu, Y. Luo and T. Ma, Inorg. Chim. Acta, 2011, 376, 619-627.

129 K. Ohta, Y. Inagaki-Oka, H. Hasebe and I. Yamamoto, Polyhedron, 2000, 19, 267-274.

130 H. Horie, A. Takagi, H. Hasebe, T. Ozawa and K. Ohta, J. Mater. Chem., 2001, 11, 1063-1071.

131 A. M. Giroud and U. T. Mueller-Westerhoff, Mol. Cryst. Liq. Cryst., 1977, 41, 11-13.

132 C. L. Kean, D. O. Miller and P. G. Pickup, J. Mater. Chem., 2002, 12, 2949-2956.

133 A. Mizuno, H. Benjamin, Y. Shimizu, Y. Shuku, M. M. Matsushita, N. Robertson and K. Awaga, Adv. Funct. Mater., 2019, 29, 1904181.

134 H. Benjamin, M. L. Müller, S. Afanasjevs, K. V. Kamenev and N. Robertson, Dalton Trans., 2020, 49, 13786-13796.

135 S. Rabaça, A. C. Cerdeira, S. Oliveira, I. C. Santos, R. T. Henriques, L. C. J. Pereira, J. T. Coutinho and M. Almeida, Polyhedron, 2012, 39, 91-98.

136 G. N. Schrauzer and V. Mayweg, J. Am. Chem. Soc., 1962, 84, 3221. 137 N. Kuramoto and K. Asao, Dyes Pigm., 1990, 12, 65-76.

138 D. Sartain and M. R. Truter, J. Chem. Soc. A, 1967, 1264-1272.

139 A. Dutta, A. Chatterjee, K. Bhar, A. Banerjee and R. Ghosh, J. Chem. Sci., 2019, 131, 1-7.

140 M. Megnamisi-Belombe and B. Nuber, Bull. Chem. Soc. Jpn., 1989, 62, 4092-4094.

141 A. Davison and E. T. Shamwl, Chem. Commun., 1967, 670.

142 A. Davison and E. T. Shawl, Inorg. Chem., 1970, 9, 1820-1825.

143 W. B. Heuer, A. E. Ture, P. N. Swepston and B. M. Hoffman, Inorg. Chem., 1988, 27, 1474-1482.

144 M. C. Aragoni, M. Arca, F. A. Devillanova, F. Isaia, V. Lippolis, A. Mancini, L. Pala, G. Verani, T. Agostinelli, M. Caironi, D. Natali and M. Sampietro, Inorg. Chem. Commun., 2007, 10, 191-194.

145 F. Bigoli, P. Deplano, F. A. Devillanova, V. Lippolis, P. J. Lukes, M. L. Mercuri, M. A. Pellinghelli and E. F. Trogu, J. Chem. Soc., Chem. Commun., 1995, 70, 371-372.

146 F. Bigoli, P. Deplano, M. Laura, M. Angela, G. Pintus, F. Trogu, G. Zonnedda, H. H. Wang and J. M. Williams, Inorg. Chim. Acta, 1998, 273, 175-183.

147 M. C. Aragoni, M. Arca, T. Cassano, C. Denotti, F. A. Devillanova, F. Isaia, V. Lippolis, D. Natali, L. Nitti, M. Sampietro, R. Tommasi and G. Verani, Inorg. Chem. Commun., 2002, 5, 869-872.

148 C. Deiana, M. Carla Aragoni, F. Isaia, V. Lippolis, A. Pintus, A. M. Z. Slawin, J. Derek Woollins and M. Arca, New J. Chem., 2016, 40, 8206-8210.

149 R. Perochon, C. Poriel, O. Jeannin, L. Piekara-Sady and M. Fourmigué, Eur. J. Inorg. Chem., 2009, 5413-5421.

150 A. Famengo, D. Pinero, O. Jeannin, T. Guizouarn, L. PiekaraSady and M. Fourmigué, New J. Chem., 2012, 36, 638-643. 